\title{
Fabrication of size-controlled metallic nanogaps down to the sub 3-nm level
}

\author{
Sihai Luo ${ }^{1}$, Andrea Mancini ${ }^{2}$, Rodrigo Berté ${ }^{2}$, Bård H. Hoff ${ }^{1}$, Stefan A. Maier ${ }^{2,3}$ and John C. deMello ${ }^{1 *}$ \\ ${ }^{1}$ Dept. Chemistry, Norwegian University of Science and Technology (NTNU), \\ NO-7491 Trondheim, Norway \\ ${ }^{2}$ Nano-Institute Munich, Faculty of Physics, Ludwig-Maximilians-Universität München, 80539 \\ München, Germany \\ ${ }^{3}$ Blackett Laboratory, Department of Physics, Imperial College London, \\ London SW7 2AZ, UK
}

*john.demello@ntnu.no

\begin{abstract}
Metallic nanogaps are fundamental components of nanoscale photonic and electronic devices. However, the lack of reproducible high-yield fabrication methods with nanometric control over the gap-size has hindered practical applications. Here, we report a patterning technique based on molecular self-assembly and physical peeling that allows the gap-width to be tuned over the range 3 - $30 \mathrm{~nm}$ and enables the fabrication of massively parallel nanogap arrays containing hundreds of millions of ring-shaped nanogaps (RSNs). The method is used here to prepare molecular diodes across sub-3-nm metallic nanogaps and to fabricate visible-light-active plasmonic substrates based on largearea, gold-based RSN arrays. The substrates are applicable to a broad range of optical applications, and are used here as substrates for surface-enhanced Raman spectroscopy (SERS), providing high enhancement factors of up to $3 \times 10^{8}$ relative to similar, gap-free thin gold films.
\end{abstract}

\section{Introduction}

Laterally aligned metal electrodes, separated on the nanometre length scale, are essential elements of many nanoscale photonic and electronic devices ${ }^{1,2}$. The small gap widths make them ideal choices for all-electronic biosensors, with the capture of a single biomolecule within or across the metallic nanogap (MNG) leading to large measurable changes in the electrical characteristics ${ }^{3-5}$. MNGs are essential components of molecular electronic devices, where conductive molecules are attached across the gap (individually or in groups) and serve as functional semiconductors in highly miniaturised rectifiers, switches and transistors ${ }^{1,6}$. They also permit the manipulation of light via plasmonic interactions, with illumination of the nanogap inducing resonant oscillations of the free electrons inside the metal electrodes (surface plasmon polaritons) ${ }^{7-11}$. The oscillating electrons act as electric dipoles that re-emit light coherently at the same frequency as the incident radiation, and additionally allow for channelling of a significant fraction of electromagnetic energy from the far field to highly confined near-field regions within the nanogaps. These optical near fields can be many orders of magnitude greater than that of the incoming light, allowing the nanogaps to act as highly localised sources of light, heat or energetic electrons for e.g. photocatalysis ${ }^{12,13}$, surface-enhanced spectroscopy $y^{14-16}$, nano-lasers ${ }^{17,18}$, solar cells $s^{19,20}$ and plasmonic circuits ${ }^{21,22}$.

Nanogap devices promise to revolutionise numerous aspects of modern science and technology, but they are currently being held back by the absence of fast, controlled, reliable (high yield) and low cost methods of fabricating MNGs with electrode spacings below $10 \mathrm{~nm}$. Typical fabrication methods entail 
the use of e-beam lithography $(E B L)^{23-25}$, mechanical break junctions $s^{6,26,27}$, electrochemical migration ${ }^{28-30}$, atomic layer lithography ${ }^{31-34}$, or focused-ion beam (FIB) milling ${ }^{35,36}$ to create the nanogaps. Such methods, however, variously suffer from low throughput, poor scalability to large substrate sizes, complex multi-step processing protocols, and/or high equipment costs. In addition, most techniques are limited to the patterning of a single metal, and so cannot be applied to the fabrication of asymmetric nanoscale devices based on dissimilar metals. The most advanced technique for patterning at the sub-10-nm level is extreme UV lithography (EUVL), which extends optical projection lithography to UV wavelengths of $\sim 13.5 \mathrm{~nm} .{ }^{37,38}$ Still in its early stages of commercialisation, EUVL is available to very few researchers and as yet cannot access feature sizes below $5 \mathrm{~nm}$ or asymmetric device structures.

In several recent reports ${ }^{36,39-46}$, it has been shown that peeling-based methods - which use surface modification to spatially control the adhesion of a deposited metal to underlying features on a substrate - provide a simple means of fabricating nanogap electrodes. One particularly effective peeling-based technique known as adhesion lithography (or "a-lith") uses self-assembled monolayers (SAMs) as adhesion modifiers, allowing MNGs with gap-widths as low as $10 \mathrm{~nm}$ to be fabricated using a small number of simple processing steps and inexpensive equipment. ${ }^{47,48,49-58}$ Until now, the reported gap-widths obtained by a-lith have been much higher than the 2-nm length of the SAM molecule, which is presumed to determine the absolute resolution limit of the technique. Hence, it has not been possible to apply adhesion lithography to applications that require the control of materials properties or light-matter interactions at extreme (sub-5-nm) length-scales, e.g. molecular electronics and plasmonics. In addition, there have been no reported methods for systematically tuning the width of the nanogaps or for fabricating massively parallel arrays of identical nanogap structures (as e.g. required for surface-enhanced spectroscopy or catalysis ${ }^{10}$ ).

In this paper, we introduce a new form of adhesion lithography that uses multilayer adhesion modifiers formed from metal-ligated self-assembled monolayers ("molecular rulers") to improve the resolution, control and versatility of the a-lith procedure. The new procedure - which we call sizetuneable adhesion lithography (STAL) - allows the gap-width to be tuned over the range $3-30 \mathrm{~nm}$ (with a resolution of a few $\mathrm{nm}$ ), and can be used to fabricate massively parallel nanogap arrays containing hundreds of millions of ring-shaped nanogaps (RSNs) of controllable diameter, width and pitch. Importantly size-tuneable adhesion lithography retains all the advantages of conventional adhesion lithography - namely it involves only a few simple processing steps that can be carried out at room temperature under ambient conditions, it uses inexpensive equipment, it can be used to fabricate nanogaps of arbitrary shape formed from dissimilar metals, and it can be applied over large areas $\left(>1 \mathrm{~cm}^{2}\right)$ - while at the same-time providing control over the gap-width down to the $3 \mathrm{~nm}$ level.

\section{Results and Discussion}

The adhesion lithography procedure is shown schematically in Fig. 1. In its usual form, a first metal (M1) is patterned on a substrate (Fig. 1a), and conformally coated with an adhesion modifier such as an alkyl-functionalized self-assembled monolayer (Fig. 1b), rendering it non-adhesive to other metals; a second metal (M2) of equal height is then deposited uniformly over the full area of the substrate (Fig. 1c); finally, the parts of the second metal that are in contact with the adhesion modifier are stripped away using an adhesive tape or film (Fig. 1d), leaving the first and second metals side-by-side on the substrate, with a self-assembled monolayer between them. ${ }^{47,48}$ Treatment with oxygen plasma or UV/ozone removes the self-assembled monolayer, resulting in a metallic nanogap (Fig. 1e).

Pre-fracturing the second metal: The first step towards achieving size-tuneability is to ensure the gapwidth attained in conventional a-lith is as close as possible to the length of the adhesion modifier, around $2 \mathrm{~nm}$ in the case of the 1-octadecanethiol (ODT) SAM molecules used here ${ }^{59}$. Successful patterning by a-lith requires (Fig. 1, step (d)) the (unwanted) parts of the second metal (M2) that lie above the SAM to be "split" from the (required) parts of M2 that are in direct contact with the 
substrate; the former must be lifted away by the peeling layer, while the latter must remain on the substrate. To effect the split, it is necessary to overcome any cohesive forces that exist between the M2 metal atoms at the intended "break-lines", i.e. along the edge profile of M1. Any tearing (as opposed to clean splitting) of M2 during peeling risks widening the gap-width undesirably. Interestingly, imaging of the surface of freshly-deposited M2 by atomic force microscopy (AFM) has previously revealed the existence of fracture lines that follow the edge profile of $M 1$, suggesting the necessary split has partially occurred even before the peeling step is carried out ${ }^{47}$. In this work, to further promote this pre-fracturing of $\mathrm{M} 2$, we use a staggered geometry in which we deliberately introduce a height difference between the two metals, with $\mathrm{M} 2$ being appreciably thinner than $\mathrm{M} 1-$ $30 \mathrm{~nm}$ versus $50 \mathrm{~nm}$ for the work reported here. The thin M2 layer is unable to conformally coat the terrain of the M1-patterned substrate, and discontinuities in $\mathrm{M} 2$ therefore arise along the edge profile of M1. In other words, the height difference between the two metals causes M2 to split exactly where the wanted and unwanted parts must be separated during the peeling step, ensuring a clean peel without tearing.

Figs. 2a,b shows scanning electron microscopy (SEM) images of Au-Al nanogaps fabricated under equivalent conditions, using matched and unmatched metal heights for M1 and M2. Octadecanethiol was used as the SAM, Au as the first metal and Al as the second metal (see Methods for fabrication details). Using a film thickness of $50 \mathrm{~nm}$ for both metals yielded a gap-width of approximately $20 \mathrm{~nm}$ at the Au/Al interface (Fig. 2a), typical of a-lith gap-widths reported elsewhere in the literature. (Note to ease visualisation of the nanogap, the image contrast in Fig. 2a has been enhanced using contrastlimited adaptive histogram equalization. The original unprocessed image may be seen in Fig. S1). Reducing the Al thickness to $30 \mathrm{~nm}$ resulted in a much narrower gap between the metals below the 3-nm resolution limit of the microscope due to pre-fracturing of $M 2$ before peeling (see Fig. $2 \mathrm{~b}$ ). Fig. 2c shows an SEM image of an $\mathrm{Au}(50-\mathrm{nm}) / \mathrm{Al}(30-\mathrm{nm})$ nanogap measured on a high-resolution scanning electron microscope. The small $10 \mathrm{~nm} \times 10 \mathrm{~nm}$ region denoted by the yellow square in Fig. $2 \mathrm{c}$ is reproduced at higher magnification in Fig. $2 \mathrm{~d}$. The images clearly show a very small gap between the two metals, less than $3 \mathrm{~nm}$ in width and close to the minimum electrode spacing that can be expected using the 2-nm ODT molecule.

Fig. 2e shows a transmission microscope (TEM) image of the gap. As shown schematically in Fig. $2 f$, the upper- and lower-most parts of the SEM image correspond to the $\mathrm{SiO}_{2}$ substrate and a platinumbased protection layer used during sample preparation (see Methods). The light region on the left between the $\mathrm{SiO}_{2}$ and $\mathrm{Pt}$ corresponds to $\mathrm{Al}$, while the dark region on the right corresponds to $\mathrm{Au}$. At the interface between the $\mathrm{Al}$ and $\mathrm{Au}$, a thin strip of $\mathrm{Pt}$ is visible (see inside dotted yellow box), corresponding to evaporated $\mathrm{Pt}$ that has penetrated the gap between the two electrodes. The width of this strip indicates a sub-3-nm gap-width in broad agreement with the SEM image in Fig. 2c.

Controlling the length of the adhesion modifier. The original a-lith procedure provides no method for tuning the electrode spacing, with the obtained gap-widths typically being anywhere from five to fifty times larger than the 2-nm length of the SAM molecule (depending for instance on how aggressively the peeling step is carried out $)^{47,48}$. In the previous section, we discussed how introducing a 20-nm height difference between the two metals permits a clean split of $M 2$ along the edge profile of M1 and so yields a gap-width close to the 2-nm length of the SAM molecule. This suggests that, using a staggered geometry, it should be possible to tune the electrode spacing simply by changing the length of the SAM molecule or, more generally, by changing the length of the adhesion modifier.

To test this idea we replaced the SAMs used in standard a-lith by extendable chains of metal-ligated self-assembled multilayers, known as molecular rulers ${ }^{60,61}$. The self-assembled multilayers are formed using SAM molecules with thiol head groups and carboxylic acid end groups by alternately immersing a gold-coated substrate in ethanolic solutions of the SAM molecules and copper perchlorate (see Fig. 1f-k and Methods). In the first step - using Au for M1 - the thiol SAM molecules are conformally attached to the patterned gold, with the carboxylic acid groups facing outwards (Fig. 1g). In the second step, $\mathrm{Cu}(\mathrm{II})$ ions coordinate with the carboxylic acid groups of the first self-assembled monolayer, 
forming an atomically thin layer (Fig. 1h) that serves as a linker upon which a second thiol SAM may be conformally attached (Fig. 1i). With each cycle, an additional SAM is added to the multilayer, increasing the layer thickness by approximately $2 \mathrm{~nm}$, which makes it possible to vary the length of the spacer molecules from a few nanometres to several tens of nanometres in 2 -nm increments. ${ }^{60}$

In practice, the presence of outwardly facing carboxylic acid groups on the final layer of the molecular ruler causes unwanted interactions with the second metal (M2), preventing clean splitting and leaving unwanted "spots" of M2 on top of M1. Hence, in the final step of the multilayer deposition (Fig. 1k), we replace the acid-functionalised SAM with an alkyl SAM, resulting in a lipophilic surface that will not bind with the second metal. The significant difference in the surface properties of the two SAMs is evident in Figs. $1 \mathrm{l}$ and $1 \mathrm{~m}$, with the alkyl-functionalised SAM (ODT) having a substantially higher water contact angle than the acid-functionalised SAM (102 vs 46 degrees), leading to weaker interaction with the polar surface of the evaporated aluminium. (A schematic showing the complete a-lith procedure using molecular rulers is shown in Fig. S2, while Fig. S3 shows how the choice of an acid- or alkyl-containing top layer affects the reliability of the patterning procedure).

Figs. 3a-d show $500 \mathrm{~nm} \times 500 \mathrm{~nm}$ SEM images for nanogap electrodes obtained using molecular rulers formed from $N=1,5,10$ and 16 repeat units (after removal of the self-assembled multilayer). In each case, the uppermost SAM layer was an alkyI SAM, while all other layers were acid functionalised. The SEM images reveal a clear increase in the spacing between the Au (left) and the Al (right) as the number of repeat units increases, consistent with the increasing length of the molecular spacers. Histograms showing the distribution of electrode spacings along the gap are shown adjacent to each image in Figs. 3e-h, while a plot of mean gap-width $\Delta x$ versus $N$ is shown in Fig. 3i. The mean gapwidth can be seen to vary from $2.8 \mathrm{~nm}$ for $N=1$ to $30.7 \mathrm{~nm}$ for $N=16$, with the relative standard deviation (defined as the ratio of the standard deviation to the mean) falling from $\sim 40 \%$ at $2.8 \mathrm{~nm}$ to $\sim 14 \%$ at $30.7 \mathrm{~nm}$. Hence, using molecular rulers, it is possible to controllably vary the gap-width over a range of sizes that are relevant for multiple applications in molecular sensing, molecular electronics and plasmonics. ${ }^{5}$

To evaluate the feasibility of using the nanogap electrodes for molecular devices, arrays of squareshaped nanogap electrodes were fabricated using Au for the first metal (M1) and either Au or Al for the second metal (M2), see Methods. The first metal was deposited as a grid of $1 \mathrm{~mm}^{2}$ squares, and the size-tuneable a-lith method was then applied, using an adhesion modifier formed from either one $(N=1)$ or two $(N=2)$ layers of SAM molecules, yielding square nanogaps of side-length $1 \mathrm{~mm}$ (Fig. $4 \mathrm{a})$ and respective widths $\sim 3$ or $\sim 6 \mathrm{~nm}$. To complete the molecular diodes, a monolayer of the widely studied molecular conductor 11-ferrocenyl-1-undecanethiol $(\mathrm{FcC} 11)^{62-64}$ was deposited on the nanogap electrodes by immersing the electrodes overnight in a $5 \mathrm{mM}$ solution of $\mathrm{FcC1} 1$ in ethanol (see Methods).

The current-voltage $(I-V)$ characteristics of the nanogap electrodes before FcC11 deposition were measured in the bias range $-1 \mathrm{~V}$ to $+1 \mathrm{~V}$, where forward bias corresponds to the second deposited metal $(M 2=A u$ or Al) being positively biased with respect to the first deposited metal $(M 1=A u)$. The $N=1$ nanogap electrodes exhibited approximately linear current-voltage characteristics with high impedances of around 20 and $50 \mathrm{G} \Omega$ for $\mathrm{Au} / \mathrm{Au}$ and $\mathrm{Au} / \mathrm{Al}$, respectively (see dotted blue lines in Figs. $4 \mathrm{~b}, \mathrm{c})$. In both cases the $N=2$ nanogap electrodes exhibited currents below the $\sim 10 \mathrm{pA}$ detection limit of the measurement, indicating extremely high impedances in excess of $100 \mathrm{G} \Omega$ (see dotted blue lines in Figs. 4d,e). Hence, there is good electrical (and physical) isolation between the electrodes in all cases, with the $N=1$ electrodes allowing a small measurable current to flow as a consequence of through-air tunnelling and/or possible occasional shorts between the electrodes.

The $N=1$ nanogaps exhibited substantially higher currents after deposition of the FcC11 monolayer (see solid red lines in Figs. 4b, c), indicating the FcC11 molecules are able to provide a conductive bridge between the two electrodes, with the molecules forming a covalent Au-S bond at one electrode and a van der Waals type interaction at the other ${ }^{62}$. Over the $-1 \mathrm{~V}$ to $+1 \mathrm{~V}$ bias range tested, the 
measured current is due to the transport of holes through the HOMO level of FcC11 ${ }^{62,64}$. The $\mathrm{Au} / \mathrm{Au}$ devices exhibited near-symmetric $I$ - $V$ characteristics with a rectification ratio $R=|I(-1 \mathrm{~V}) / I(+1 \mathrm{~V})|$ of approximately 1.1, while the $\mathrm{Al} / \mathrm{Au}$ devices exhibited asymmetric characteristics with a rectification ratio of approximately 6.5. The broadly symmetric current-voltage characteristics of the Au/Au devices are consistent with the use of identical metals for the two electrodes, and the ability of the thiol group on the FcC11 molecules to attach to both the first (M1) and second (M2) electrodes, which has the effect of averaging out effects due to the asymmetry in the molecular structure. The asymmetric current-voltage characteristics of the Au/Al devices by contrast are attributable both to the use of dissimilar metals for the two electrodes and to the thiol head groups attaching only to M1 ( $\mathrm{Au}$ ), which preserves the effects of asymmetry in the molecular structure.

The solid red lines in Fig. $4 \mathrm{~d}$,e show for $\mathrm{Au} / \mathrm{Au}$ and $\mathrm{Au} / \mathrm{Al}$ the measured-current voltage characteristics of the $N=2$ nanogap devices after application of the FcC11 molecules. In both cases, the currents remained below the $10 \mathrm{pA}$ detection limit of the ammeter over the entire bias range (from $-1 \mathrm{~V}$ to $+1 \mathrm{~V}$ ), indicating the FcC11 molecules were unable to conductively bridge the gap. For $N=2$, we therefore conclude that the gap-width is higher than the 2.2-nm length of the FcC11 molecules over the full 4-mm length of the gap. On the other hand, for the FcC11 molecules to provide a conductive bridge between the electrodes, the $N=1$ gap-width must be less than $2.2 \mathrm{~nm}$ at many locations along the gap in agreement with the SEM image of Fig. 2c.

Massively parallel nanogap arrays. One favourable feature of a-lith is its scalability to large areas (see e.g. Fig. S4), which opens up the possibility of fabricating massively parallel arrays of nanogaps. As a proof of concept, we fabricated large-area $\left(\sim 50 \mathrm{~mm}^{2}\right)$ arrays of nanorings by combining a-lith with a soft colloidal lithography method known as nanosphere lithography (NSL). ${ }^{65}$ In brief, the combined technique comprised the following steps: a hexagonal close-packed monolayer of polystyrene (PS) spheres (Fig. 5a) was deposited on a substrate by drop-casting; the nanospheres were then isotropically "shrunk" via oxygen plasma etching so they no longer touched (Fig. 5b); the first metal was evaporated onto the substrate through the spaces between the spheres; and the spheres were then removed, leaving the first metal patterned with a hexagonal array of circular holes (see Fig. 5c and S5a); continuing the size-tuneable a-lith method from this point yielded a macroscopic array of near-identical ring-shaped nanogaps (RSNs, see Fig. $5 \mathrm{~d}$ and Fig. S6), in which the pitch, diameter and width of the RSNs were determined by the initial diameter of the PS spheres, the etched diameter of the PS spheres, and the number of layers used in the molecular ruler.

For a typical drop-cast area of $50 \mathrm{~mm}^{2}$ and an initial nanosphere diameter of around $500 \mathrm{~nm}$, each array contains some 200 million discrete and nominally identical nanorings (see Fig. S7). Like any method based on nanosphere lithography, defects such as dislocations and vacancies in the initial nanosphere template introduce disorder in the final pattern. However, executed with care, the combined NSL/STAL method provides a simple means of rapidly fabricating well-ordered and massively parallel arrays of nearly identical nanogaps that extend over multi-millimetre length-scales with relatively low defect densities, see e.g. Fig. 5e. Fig. 5f-h shows high magnification SEM images of $\mathrm{Au} / \mathrm{Au}$ RSN arrays obtained using a pitch of $\sim 500 \mathrm{~nm}$, a ring diameter of $\sim 380 \mathrm{~nm}$, and $N=1,2$ or 5 layers in the molecular ruler. Clear gaps of approximately 5 and $10 \mathrm{~nm}$ are evident in the SEM images for the 2- and 5-layer arrays, while the gap-width of the 1-layer device is below the 3-nm image resolution.

It is well known that regular arrays of sub-wavelength holes or slits in metal films allow optical energy to be efficiently coupled into surface plasmon polaritons (SPPs) - electromagnetic excitations that propagate in a wave-like manner along the planar interface between a metal and a dielectric - or into localized surface plasmons. ${ }^{8-10}$ The confinement of the electromagnetic wave to the vicinity of the metal/dielectric interface results in a substantial enhancement of the electromagnetic field and accounts for many useful surface-enhanced optical properties, e.g. increased absorption, fluorescence, Raman scattering, second-harmonic generation and chiroptical behaviour ${ }^{7,8,10,14}$. The RSN arrays are therefore of potential interest for a variety of plasmonic applications. 
To test the suitability of the RSN arrays for one such plasmonic application, their performance as Surface-Enhanced Raman Spectroscopy (SERS) substrates was evaluated, alongside a thin gold coating of similar thickness and a nanohole array (see Fig. S5a) obtained by terminating the RSN fabrication procedure after step (c) in Fig. 5, i.e. before deposition of the second metal (M2). A $10^{-4} \mathrm{M}$ solution of the test-dye Rhodamine $6 \mathrm{G}$ was drop-cast on each of the five substrates, and Raman spectra were recorded under equivalent conditions at a probe wavelength of $633 \mathrm{~nm}$ (see Methods). The resulting Raman spectra are shown in the upper plot of Fig. 6a. In spite of the high dye concentration, the thin Au film and nanohole array both yielded weak Raman spectra, with the characteristic Raman spectra barely visible above the noise floor of the measurements. The three RSN-arrays by contrast yielded well-defined spectra typical of Rhodamine 6G, with the scattering intensity increasing approximately two-fold as $N$ was reduced from 5 to 1.

Fig. $6 \mathrm{~b}$ shows a simulation of the square of the electromagnetic field enhancement at the top surface of the RSN array. To simulate the array we used periodic boundary conditions for a hexagonal lattice with $a=\sqrt{3} P_{x} / 2$, where $P_{x}=500 \mathrm{~nm}$ is the $x$ dimension of the unit cell and $a$ is the apothem of the hexagon. The source employed in the simulations was a linearly polarized plane wave at $\lambda=$ $633 \mathrm{~nm}$, matching the wavelength used for the SERS experiments. The gap-size was set to $\Delta r=3 \mathrm{~nm}$, representing the case $N=1$. The simulation shows high field intensity in the gap region with a spatially-averaged field enhancement $\left|\mathrm{E} / \mathrm{E}_{0}\right|^{2}$ of 335 , leading to high SERS enhancement for the molecules within the gap. This enhancement is achieved even though we are not operating at the exact localized plasmon resonance condition (which occurs at $\lambda=626 \mathrm{~nm}$, see Fig S8). Indeed, a key advantage of the nanoring geometry is that the resonance peaks are very broad, which means appreciable field enhancement can be achieved without needing to precisely tune the excitation wavelength to the peak of the plasmon resonance. ${ }^{66,67}$

The lower plot of Fig. 6a shows a series of Raman spectra obtained using $N=1$ RSN arrays at various dye concentrations from $10^{-6} \mathrm{M}$ down to $10^{-14} \mathrm{M}$. The spectra all show the characteristic Raman scattering peaks of Rhodamine $6 \mathrm{G}$, albeit with a reduction in scattering intensity as the dye concentration is reduced. The intensity of the strongest $633 \mathrm{~nm}$ peak versus dye concentration is shown for illustrative purposes in Fig. 6c. The strongly sub-linear variation of scattering intensity with dye concentration is consistent with previous reports in the literature using plasmonic substrates. ${ }^{26,68,69}$ Even at the lowest concentration of $10^{-14} \mathrm{M}$, most of the Raman peaks of Rhodamine $6 \mathrm{G}$ are evident. Assuming the dye molecules are randomly distributed on the RSN array and that they can couple to localised electric fields only if their centres lie within the nanogap, a purely geometric argument indicates that the measured scattering signal at $10^{-14} \mathrm{M}$ is due to around 1000 dye molecules (see Fig. S7). Comparing the $613 \mathrm{~cm}^{-1}$ signal at $10^{-14} \mathrm{M}$ with the signal obtained under equivalent conditions on a thin gold film at $10^{-5} \mathrm{M}$ (the lowest concentration at which a signal could be measured, see Fig. S9) indicates an analytical enhancement factor ${ }^{70}$ of $3 \times 10^{8}$, one of the highest values so far reported for Rhodamine $6 \mathrm{G}$ on a SERS substrate even though the $633 \mathrm{~nm}$ excitation wavelength was not tuned to be in exact resonance with the nanogap array.

\section{Conclusion}

In conclusion we have reported methods for improving the resolution, control and versatility of the alith procedure. By introducing a height difference of around $20 \mathrm{~nm}$ between the first and second metals we were able to achieve a substantial narrowing of the achievable gap-width to below 3-nm - only slightly higher than the 2-nm length of the SAM molecule, and sufficiently small for the fabrication of molecular rectifiers using the molecular conductor 11-ferrocenyl-1-undecanethiol, FcC11. Further, by replacing the fixed-length SAMs by variable-length chains of carboxylic acid functionalised SAM molecules ("molecular rulers"), we showed that it is possible to vary the gap-width over the range 2$30 \mathrm{~nm}$. Finally, by combining a-lith with nanosphere lithography - i.e. using a hexagonal array of 
etched nanospheres as a sacrificial shadow mask for deposition of the first metal - we fabricated massively parallel arrays of ring-shaped nanogaps (RSNs) of variable diameter, width and pitch. The arrays - which extend over millimetre length-scales and contain hundreds of millions of ring-shaped nanogaps - strongly confine electromagnetic fields under visible light illumination, making them suitable for a range of plasmonic applications. The RSN arrays were evaluated here as substrates for surface-enhanced Raman spectroscopy, using Rhodamine $6 \mathrm{G}$ as a test molecule, and were found to exhibit very high enhancement factors of $3 \times 10^{8}$ relative to a similar (gap-free) thin gold film. 


\section{Methods}

Fabrication of single-layer metal nanogaps: A $5 \mathrm{~nm}$ adhesion layer of Al followed by a $45 \mathrm{~nm}$ layer of $\mathrm{Au}(\mathrm{M} 1)$ was deposited on a glass substrate, using e-beam deposition via a shadow mask $\left(10^{-7} \mathrm{mbar}\right.$, $2 \AA \mathrm{s}^{-1}$ ). The substrate was then immersed in a $2 \mathrm{mM}$ ethanolic solution of ODT for $24 \mathrm{~h}$, before washing thoroughly in clean ethanol. A $30 \mathrm{~nm}$ layer of Al or Au (M2) was then deposited by e-beam deposition over the full area of the metal-coated substrate $\left(10^{-7} \mathrm{mbar}, 2 \AA^{-1}\right)$. An adhesive film (First Contact Red, Photonic Cleaning Technologies) was drop-cast on top of the substrate, allowed to dry at room temperature, and then peeled away by hand, leaving $\mathrm{M} 1$ and $\mathrm{M} 2$ in a side-by-side arrangement and separated by an ODT monolayer. In the final step, the monolayer was removed by $\mathrm{O}_{2}$ plasma treatment for $3 \mathrm{~min}$ ( $100 \mathrm{~W}, \mathrm{O}_{2}$ flow rate: $5 \mathrm{~mL} \mathrm{~min}{ }^{-1}$ ).

Fabrication of multi-layer metal nanogaps: Fabrication was carried out using an equivalent procedure to the one used for the single-layer metal nanogaps, except the ODT monolayer was replaced by a molecular ruler, i.e. a metal-ligated multilayer. The molecular rulers were prepared according to a literature protocol ${ }^{65}$ by first immersing the substrate in a $2 \mathrm{mM}$ ethanolic solution of 16 mercaptohexadecanoic acid (MHDA) for $12 \mathrm{~h}$ to form a densely packed monolayer on top of M1 (Au). Further layers of MHDA were then added in a step-wise manner by alternately immersing the substrate in a $2 \mathrm{mM}$ ethanolic solution of copper perchlorate for $15 \mathrm{~min}$ and a $2 \mathrm{mM}$ ethanolic solution of MHDA for $30 \mathrm{~min}$, washing thoroughly in clean ethanol between each process step. In the final step of the multilayer preparation (after $\mathrm{Cu}\left(\mathrm{ClO}_{4}\right)_{2}$ treatment), the substrate was immersed in a $2 \mathrm{mM}$ solution of ODT in ethanol for $24 \mathrm{~h}$, yielding an upper surface of non-reactive alkyl groups in the molecular ruler. From this point onwards the fabrication procedure was identical to the single-layer procedure.

Fabrication of Ring-shaped Nanogap (RSN) arrays: RSN arrays were fabricated according to the singleand multi-layer nanogap procedures described above, except a template of polystyrene nanospheres was used as a shadow mask for deposition of M1 and 5-nm Ti (instead of 5-nm Al) was deposited beneath the $45-\mathrm{nm}$ gold to minimise plasmonic losses. To prepare the nanosphere template, a glass substrate was sequentially cleaned with acetone, ethanol and deionized water, dried in a stream of nitrogen, and then subjected to $\mathrm{O}_{2}$ plasma for $3 \mathrm{~min}\left(100 \mathrm{~W}, \mathrm{O}_{2}\right.$ flow rate: $\left.5 \mathrm{~mL} \mathrm{~min}^{-1}\right)$. A circular well of polydimethylsiloxane (PDMS) of diameter $1 \mathrm{~cm}$ and height $2 \mathrm{~mm}$ was placed in the centre of the glass substrate. A $10 \mathrm{wt} . \%$ suspension of $500-\mathrm{nm}$ polystyrene (PS) nanospheres in water [Product no. 59769 , Sigma-Aldrich,] was volumetrically diluted by $50 \%$ in ethanol, and loaded into a micropipette. A $0.5 \mu \mathrm{L}$ droplet of the diluted solution was deposited inside the PDMS well, and allowed to dry under ambient conditions, yielding a close-packed monolayer of nanospheres. The nanospheres were then etched with $\mathrm{O}_{2}$ plasma ( $100 \mathrm{~W}, \mathrm{O}_{2}$ flow rate: $5 \mathrm{~mL} \mathrm{~min}^{-1}$ ) for $10 \mathrm{~min}$, causing them to shrink in volume, while remaining at their original location. A 5-nm adhesion layer of titanium, followed by a 45-nm layer of gold was deposited onto the templated substrate by e-beam evaporation $\left(10^{-7} \mathrm{mbar}, 2 \AA \mathrm{s}^{-1}\right)$. The PS nanospheres were removed using one-sided $3 \mathrm{M}$ Scotch tape, leaving behind a hexagonal array of nanoholes in the gold film. The size-controlled a-lith method was then carried out using ODT (single layer) or an ODT-terminated MHDA molecular ruler (multi-layer) as described above.

Imaging: Scanning electron microscopy (SEM) images of the nanogap electrodes were recorded on an electron microscope (FEI APREO) using an electron-beam voltage of $10 \mathrm{kV}$ and a current of $13 \mathrm{pA}$. For imaging by transmission-electron microscopy (TEM), a single-layer Au/Al nanogap was prepared using the technique described above. The nanogap was then processed into a Au/air/Al laminar using a focused ion-beam (FIB) instrument (FEl Helios) equipped with a nanomanipulator (Omniprobe, AutoProbe300) using the "lift-out" method. Electron-beam assisted carbon and platinum deposition were performed on the sample surface to protect it against the ion beam bombardment during ionbeam milling. A Ga ion-beam ( $30 \mathrm{kV}, 9 \mathrm{nA}$ ) was first used to cut the sample from the bulk, and then it was attached to a Cu grid using the lift-out method. The sample was subsequently thinned down to a thickness of approximately $90 \mathrm{~nm}(30 \mathrm{kV}, 93 \mathrm{pA})$ for imaging by S(T)EM (S-5500 model, Hitachi). Prior 
to FIB patterning, high Resolution SEM images of the nanogap were recorded on the same S(T)EM using an electron-beam voltage of $3 \mathrm{kV}$ and a current of $9 \mathrm{pA}$.

Electrical Measurements: Molecular diodes based on single and double-layer Au/Al nanogap electrodes were fabricated by following the single- and multi-layer procedures mentioned above. The organic spacers were removed by oxygen plasma cleaning for a period of $5 \mathrm{~min}\left(100 \mathrm{~W}, \mathrm{O}_{2}\right.$ flow rate: $5 \mathrm{~mL} \mathrm{~min}{ }^{-1}$ ). Next, the substrates were immersed in a $5 \mathrm{mM}$ solution of FcC11 in ethanol for $24 \mathrm{~h}$, rinsed with ethanol, and then dried under nitrogen gas. Electrical contact was made to the devices using a probe station (Micromanipulators, Imina Technologies), before testing in air with a Keithley SCS 4202 parameter analyser. An equivalent procedure was followed for Au/Au molecular diodes. Forward bias corresponded to the second deposited metal (M2 = Au or Al) being positively biased with respect to the first deposited metal $(M 1=A u)$.

Optical Measurements: To prepare the samples for SERS measurements, $5 \mu \mathrm{L}$ ethanolic solutions of R6G of varying concentration were drop-cast onto as-fabricated RSN arrays and dried in air for about $12 \mathrm{~h}$. Raman spectra were obtained on a confocal Raman spectrometer using $0.5 \mathrm{~mW}, 633 \mathrm{~nm}$ laser excitation. The laser beam was focused onto the sample through $a \times 50$ objective lens (NA=0.75), and all spectra were acquired using a $10 \mathrm{~s}$ acquisition time.

Simulations: 3D electromagnetic simulations were performed with the software package CST Studio in the frequency domain. To simulate the array, periodic boundary conditions were used in the $x, y$ directions, with a rectangular unit cell of dimensions $P_{x}=500 \mathrm{~nm}$ and $P_{y}=2 \cdot P_{x} \sin (\pi / 3)$. The unit cell has one RSN at the centre and a quarter of a RSN at each vertex. The thickness of the gold was set to $50 \mathrm{~nm}$ for $\mathrm{M} 1$ and $30 \mathrm{~nm}$ for M2. A linearly polarized plane wave at normal incidence in combination with Floquet Mode Ports was used to simulate the excitation of the $\lambda=633 \mathrm{~nm}$ laser used for the SERS experiments. The internal diameter of the RSN was $380 \mathrm{~nm}$ and the gap width was $\Delta r=3 \mathrm{~nm}$, corresponding to $N=1$. The RSN hexagonal array was simulated on a glass substrate to match the fabricated structure.

\section{Acknowledgements}

The experimental work was undertaken in the NTNU NanoLab with financial support from NTNU, project number 81771118 . The Research Council of Norway is acknowledged for support via the Norwegian Micro and Nano-Fabrication Facility, NorFab, project number 245963/F50. Partial funding for this work was obtained from the Norwegian PhD Network on Nanotechnology for Microsystems, which was sponsored by the Research Council of Norway, Division for Science, under contract no.221860/F60. The authors thank Mr. K. Hunnestad and V. Håkonsen for help with FIB milling and TEM imaging. S.A.M. acknowledges the Lee-Lucas Chair in Physics, the Bavarian Solar Energies go Hybrid Programme, and the DFG Cluster of Excellence e-conversion (EXC 2089/1-390776260).

\section{Author contributions}

S.L, B.H.H. and J.C.deM designed the experimental measurements. S.L. carried out the experiments. R.B., A.M. and S.A.M. designed and carried out the simulations. All authors analysed the data and contributed to the writing of the manuscript. 


\section{References}

1. Li, T., Hu, W. \& Zhu, D. Nanogap electrodes. Adv. Mater. 22, 286-300 (2010).

2. Dubois, V., Bleiker, S. J., Stemme, G. \& Niklaus, F. Scalable Manufacturing of Nanogaps. Adv. Mater. 1801124, 1801124 (2018).

3. Shafer-Peltier, K. E., Haynes, C. L., Glucksberg, M. R. \& Van Duyne, R. P. Toward a glucose biosensor based on surface-enhanced Raman scattering. J. Am. Chem. Soc. 125, 588-593 (2003).

4. Chen, X. et al. Electrical nanogap devices for biosensing. Mater. Today 13, 28-41 (2010).

5. Yang, Y., Gu, C. \& Li, J. Sub-5 nm Metal Nanogaps: Physical Properties, Fabrication Methods, and Device Applications. Small 15, 1-26 (2019).

6. Cui, A., Dong, H. \& Hu, W. Nanogap Electrodes towards Solid State Single-Molecule Transistors. Small 11, 6115$6141(2015)$.

7. Halas, N. J., Lal, S., Chang, W. S., Link, S. \& Nordlander, P. Plasmons in strongly coupled metallic nanostructures. Chem. Rev. 111, 3913-3961 (2011).

8. Gu, P., Zhang, W. \& Zhang, G. Plasmonic Nanogaps: From Fabrications to Optical Applications. Adv. Mater. Interfaces 5, 1-22 (2018).

9. $\mathrm{Xu}, \mathrm{Y}$. et al. Light-Matter Interaction within Extreme Dimensions: From Nanomanufacturing to Applications. Adv. Opt. Mater. 6, 1-14 (2018).

10. Bahk, Y. M., Kim, D. S. \& Park, H. R. Large-Area Metal Gaps and Their Optical Applications. Adv. Opt. Mater. 7, 121 (2019).

11. Baumberg, J. J., Aizpurua, J., Mikkelsen, M. H. \& Smith, D. R. Extreme nanophotonics from ultrathin metallic gaps. Nat. Mater. 18, 668-678 (2019).

12. Ai, B., Wang, Z., Möhwald, H. \& Zhang, G. Plasmonic Nanochemistry Based on Nanohole Array. ACS Nano 11, 12094-12102 (2017).

13. Yin, Z. et al. Hybrid Au-Ag Nanostructures for Enhanced Plasmon-Driven Catalytic Selective Hydrogenation through Visible Light Irradiation and Surface-Enhanced Raman Scattering. J. Am. Chem. Soc. 140, 864-867 (2018).

14. Langer, J. et al. Present and future of surface-enhanced Raman scattering. ACS Nano 14, 28-117 (2020).

15. Park, S. G. et al. Self-Assembly of Nanoparticle-Spiked Pillar Arrays for Plasmonic Biosensing. Adv. Funct. Mater. 29, 1-9 (2019).

16. Chen, X., Ciracì, C., Smith, D. R. \& Oh, S. H. Nanogap-enhanced infrared spectroscopy with template-stripped wafer-scale arrays of buried plasmonic cavities. Nano Lett. 15, 107-113 (2015).

17. Chou, Y. H. et al. Ultracompact Pseudowedge Plasmonic Lasers and Laser Arrays. Nano Lett. 18, 747-753 (2018).

18. Ho, J. et al. A Nanowire-Based Plasmonic Quantum Dot Laser. Nano Lett. 16, 2845-2850 (2016).

19. Ueno, K., Oshikiri, T., Sun, Q., Shi, X. \& Misawa, H. Solid-State Plasmonic Solar Cells. Chem. Rev. 118, 2955-2993 (2018).

20. Munday, J. N. \& Atwater, H. A. Large integrated absorption enhancement in plasmonic solar cells by combining metallic gratings and antireflection coatings. Nano Lett. 11, 2195-2201 (2011).

21. Koch, U. et al. A monolithic bipolar CMOS electronic-plasmonic high-speed transmitter. Nat. Electron. 3, 338-345 (2020).

22. Schörner, C., Adhikari, S. \& Lippitz, M. A Single-Crystalline Silver Plasmonic Circuit for Visible Quantum Emitters. Nano Lett. 19, 3238-3243 (2019).

23. Eklöf-Österberg, J., Gschneidtner, T., Tebikachew, B., Lara-Avila, S. \& Moth-Poulsen, K. Parallel Fabrication of SelfAssembled Nanogaps for Molecular Electronic Devices. Small 14, 1-6 (2018).

24. Flauraud, V. et al. In-Plane Plasmonic Antenna Arrays with Surface Nanogaps for Giant Fluorescence Enhancement. Nano Lett. 17, 1703-1710 (2017).

25. Duan, H., Hu, H., Kumar, K., Shen, Z. \& Yang, J. K. W. Direct and reliable patterning of plasmonic nanostructures with sub-10-nm gaps. ACS Nano 5, 7593-7600 (2011).

26. Pan, R. et al. Nanocracking and metallization doubly defined large-scale 3D plasmonic sub-10 nm-gap arrays as extremely sensitive SERS substrates. Nanoscale 10, 3171-3180 (2018). 
27. Xiang, D., Jeong, H., Lee, T. \& Mayer, D. Mechanically controllable break junctions for molecular electronics. Adv. Mater. 25, 4845-4867 (2013).

28. Johnston, D. E., Strachan, D. R. \& Johnson, A. T. C. Parallel fabrication of nanogap electrodes. Nano Lett. 7, 27742777 (2007).

29. Suga, H. et al. Feedback Electromigration Assisted by Alternative Voltage Operation for the Fabrication of FacetEdge Nanogap Electrodes. ACS Appl. Nano Mater. 3, 4077-4083 (2020).

30. Naitoh, Y. et al. Self-aligned formation of sub $1 \mathrm{~nm}$ gaps utilizing electromigration during metal deposition. ACS Appl. Mater. Interfaces 5, 12869-12875 (2013).

31. Chen, X. et al. Atomic layer lithography of wafer-scale nanogap arrays for extreme confinement of electromagnetic waves. Nat. Commun. 4, 1-7 (2013).

32. Yoo, D. et al. High-Throughput Fabrication of Resonant Metamaterials with Ultrasmall Coaxial Apertures via Atomic Layer Lithography. Nano Lett. 16, 2040-2046 (2016).

33. Yoo, D. et al. High-Contrast Infrared Absorption Spectroscopy via Mass-Produced Coaxial Zero-Mode Resonators with Sub-10 nm Gaps. Nano Lett. 18, 1930-1936 (2018).

34. Barik, A., Chen, X. \& Oh, S. H. Ultralow-Power Electronic Trapping of Nanoparticles with Sub-10 nm Gold Nanogap Electrodes. Nano Lett. 16, 6317-6324 (2016).

35. Cui, A. et al. Single grain boundary break junction for suspended nanogap electrodes with gapwidth down to 1-2 $\mathrm{nm}$ by focused ion beam milling. Adv. Mater. 27, 3002-3006 (2015).

36. Chen, Y. et al. Rapid Focused Ion Beam Milling Based Fabrication of Plasmonic Nanoparticles and Assemblies via 'sketch and Peel' Strategy. ACS Nano 10, 11228-11236 (2016).

37. Wagner, C. \& Harned, N. EUV lithography: Lithography gets extreme. Nat. Photonics 4, 24-26 (2010).

38. Tallents, G., Wagenaars, E. \& Pert, G. Optical lithography: Lithography at EUV wavelengths. Nat. Photonics 4, 809811 (2010).

39. Zheng, M. et al. Kirigami-inspired multiscale patterning of metallic structures via predefined nanotrench templates. Microsystems Nanoeng. 5, (2019).

40. Chen, Y. et al. Adhesion-Engineering-Enabled "Sketch and Peel" Lithography for Aluminum Plasmonic Nanogaps. Adv. Opt. Mater. 8, 1-8 (2020).

41. Chen, Y. et al. Reliable Patterning, Transfer Printing and Post-Assembly of Multiscale Adhesion-Free Metallic Structures for Nanogap Device Applications. Adv. Funct. Mater. 30, 1-8 (2020).

42. Chen, Y. et al. 'sketch and Peel' Lithography for High-Resolution Multiscale Patterning. Nano Lett. 16, 3253-3259 (2016).

43. Bahk, Y. M. et al. Electromagnetic Saturation of Angstrom-Sized Quantum Barriers at Terahertz Frequencies. Phys. Rev. Lett. 115, 1-5 (2015).

44. Cui, A. et al. Mass Production of Nanogap Electrodes toward Robust Resistive Random Access Memory. Adv. Mater. 28, 8227-8233 (2016).

45. Chen, X. et al. Atomic layer lithography of wafer-scale nanogap arrays for extreme confinement of electromagnetic waves. Nat. Commun. 4, 1-7 (2013).

46. Ji, D. et al. Efficient Mid-Infrared Light Confinement within Sub-5-nm Gaps for Extreme Field Enhancement. Adv. Opt. Mater. 5, 1-9 (2017).

47. Beesley, D. J. et al. Sub-15-nm patterning of asymmetric metal electrodes and devices by adhesion lithography. Nat. Commun. 5, 1-9 (2014).

48. Luo, S., Hoff, B. H. \& deMello, J. C. Spontaneous Formation of Nanogap Electrodes by Self-Peeling Adhesion Lithography. Adv. Mater. Interfaces 1900243, 1-8 (2019).

49. Wyatt-Moon, G., Georgiadou, D. G., Zoladek-Lemanczyk, A., Castro, F. A. \& Anthopoulos, T. D. Flexible nanogap polymer light-emitting diodes fabricated via adhesion lithography (a-Lith). J. Phys. Mater. 1, 01 LT01 (2018).

50. Tripathi, L. N. et al. Quantum dots-nanogap metamaterials fabrication by self-assembly lithography and photoluminescence studies. Opt. Express 23, 14937 (2015).

51. Semple, J. et al. Radio Frequency Coplanar ZnO Schottky Nanodiodes Processed from Solution on Plastic Substrates. Small 12, 1993-2000 (2016). 
52. Kumar, M. et al. Colossal Tunneling Electroresistance in Co-Planar Polymer Ferroelectric Tunnel Junctions. Adv. Electron. Mater. 6, 1-7 (2020).

53. Georgiadou, D. G. et al. High Responsivity and Response Speed Single-Layer Mixed-Cation Lead Mixed-Halide Perovskite Photodetectors Based on Nanogap Electrodes Manufactured on Large-Area Rigid and Flexible Substrates. Adv. Funct. Mater. 1901371, 1-11 (2019).

54. Kawanago, T. \& Oda, S. Control of threshold voltage by gate metal electrode in molybdenum disulfide field-effect transistors. Appl. Phys. Lett. 110, (2017).

55. Wyatt-Moon, G., Georgiadou, D. G., Semple, J. \& Anthopoulos, T. D. Deep Ultraviolet Copper(I) Thiocyanate (CuSCN) Photodetectors Based on Coplanar Nanogap Electrodes Fabricated via Adhesion Lithography. ACS Appl. Mater. Interfaces 9, 41965-41972 (2017).

56. Semple, J. et al. Large-area plastic nanogap electronics enabled by adhesion lithography. npj Flex. Electron. 2, 18 (2018).

57. Semple, J., Rossbauer, S. \& Anthopoulos, T. D. Analysis of Schottky contact formation in coplanar Au/ZnO/Al nanogap radio frequency diodes processed from solution at low temperature. ACS Appl. Mater. Interfaces 8 , 23167-23174 (2016).

58. Semple, J., Wyatt-Moon, G., Georgiadou, D. G., McLachlan, M. A. \& Anthopoulos, T. D. Semiconductor-Free Nonvolatile Resistive Switching Memory Devices Based on Metal Nanogaps Fabricated on Flexible Substrates via Adhesion Lithography. IEEE Trans. Electron Devices 64, 1973-1980 (2017).

59. Walczak, M. M., Chung, C., Stole, S. M., Widrig, C. A. \& Porter, M. D. Structure and Interfacial Properties of Spontaneously Adsorbed n-Alkanethiolate Monolayers on Evaporated Silver Surfaces. J. Am. Chem. Soc. 113, 2370-2378 (1991).

60. Hatzor, A. \& Weiss, P. S. Molecular rulers for scaling down nanostructures. Science (80-. ). 291, 1019-1020 (2001).

61. Evans, S. D., Ulman, A., Goppert-Berarducci, K. E. \& Gerenser, L. J. Self-Assembled Multilayers of $\omega$ Mercaptoalkanoic Acids: Selective Ionic Interactions. J. Am. Chem. Soc. 113, 5866-5868 (1991).

62. Nijhuis, C. A., Reus, W. F., Barber, J. R., Dickey, M. D. \& Whitesides, G. M. Charge transport and rectification in arrays of SAM-based tunneling junctions. Nano Lett. 10, 3611-3619 (2010).

63. Yuan, L., Jiang, L., Thompson, D. \& Nijhuis, C. A. On the remarkable role of surface topography of the bottom electrodes in blocking leakage currents in molecular diodes. J. Am. Chem. Soc. 136, 6554-6557 (2014).

64. Jeong, H. et al. Redox-induced asymmetric electrical characteristics of ferrocene- alkanethiolate molecular devices on rigid and flexible substrates. Adv. Funct. Mater. 24, 2472-2480 (2014).

65. Lee, S. H., Bantz, K. C., Lindquist, N. C., Oh, S. H. \& Haynes, C. L. Self-assembled plasmonic nanohole arrays. Langmuir 25, 13685-13693 (2009).

66. Lin, L. et al. Electron Transport Across Plasmonic Molecular Nanogaps Interrogated with Surface-Enhanced Raman Scattering. ACS Nano 12, 6492-6503 (2018).

67. Im, H., Bantz, K. C., Lindquist, N. C., Haynes, C. L. \& Oh, S. H. Vertically oriented sub-10-nm plasmonic nanogap arrays. Nano Lett. 10, 2231-2236 (2010).

68. Im, H. et al. Self-assembled plasmonic nanoring cavity arrays for SERS and LSPR biosensing. Adv. Mater. 25, 26782685 (2013).

69. Jin, B., He, J., Li, J. \& Zhang, Y. Lotus Seedpod Inspired SERS Substrates: A Novel Platform Consisting of 3D Sub-10 nm Annular Hot Spots for Ultrasensitive SERS Detection. Adv. Opt. Mater. 6, 1-8 (2018).

70. Le Ru, E. C., Blackie, E., Meyer, M. \& Etchegoint, P. G. Surface enhanced raman scattering enhancement factors: A comprehensive study. J. Phys. Chem. C 111, 13794-13803 (2007). 

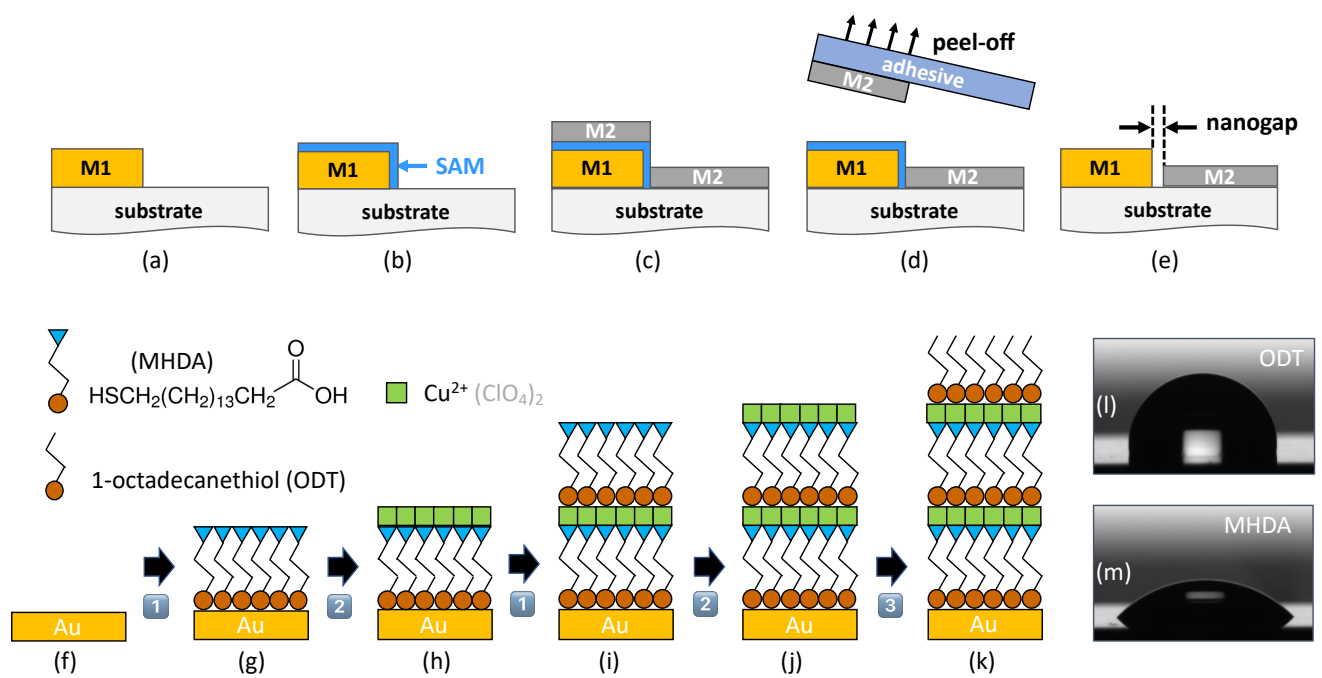

Figure 1. Schematics showing the key processing steps for patterning nanogaps by a-lith (a-e) and for forming sizecontrollable self-assembled multilayers (f-k). The a-lith procedure comprises the following steps: first, metal M1 is deposited on a substrate and patterned as appropriate (a); second, M1 is selectively coated with a metallophilic SAM (b); third, metal M2 is deposited uniformly over M1 and the exposed substrate (c); fourth, an adhesive film is applied to the surface of $M 2$ and the tape is then peeled away from the substrate, selectively removing $M 2$ from those regions located directly above the SAM (d); finally, the SAM is removed by UV/ozone or oxygen-plasma treatment, leaving M1 and M2 sitting in a complementary arrangement side-by-side on the substrate (e), separated in the limiting case by the length of the SAM. In conventional a-lith $\mathrm{M} 1$ and $\mathrm{M} 2$ have a similar thickness, while in size-controlled a-lith $\mathrm{M} 2$ is deliberately made substantially thinner than $\mathrm{M} 1$ to induce fracturing of $\mathrm{M} 2$ along the edge-profile of $\mathrm{M} 1$ (resulting in a much cleaner peel). Furthermore, in size-controlled a-lith, the SAM is replaced by a self-assembled multilayer (or "molecular ruler") formed from parallel chains of SAM molecules. The multilayer is formed by alternately immersing a gold-coated substrate (f) in ethanolic solutions of MHDA (Process 1) and copper perchlorate (Process 2), rinsing thoroughly in clean ethanol between each step. The first application of Process 1 yields a densely packed monolayer of MHDA (g), while the first application of Process 2 yields a layer of $\mathrm{CU}$ (II) ions on top of the MHDA that serves as an atomically thin linker on which a second thiol SAM may be attached. Repeating the two process steps adds another SAM to the assembly $(i, j)$, increasing the layer thickness by approximately $2 \mathrm{~nm}$. In the final step the substrate is immersed in an ethanolic solution of octadecane thiol (Process 3), yielding an alkylcapped upper layer (k). The alkyl-functionalised SAM has a much higher water contact angle [102 degrees, (I)] than the acidfunctionalised SAM [46 degrees, $(\mathrm{m})$ ], leading to weaker interaction with the polar surface of the second metal and hence easier peeling. A schematic of the size-tuneable adhesion lithography procedure is shown in Fig. S2. 


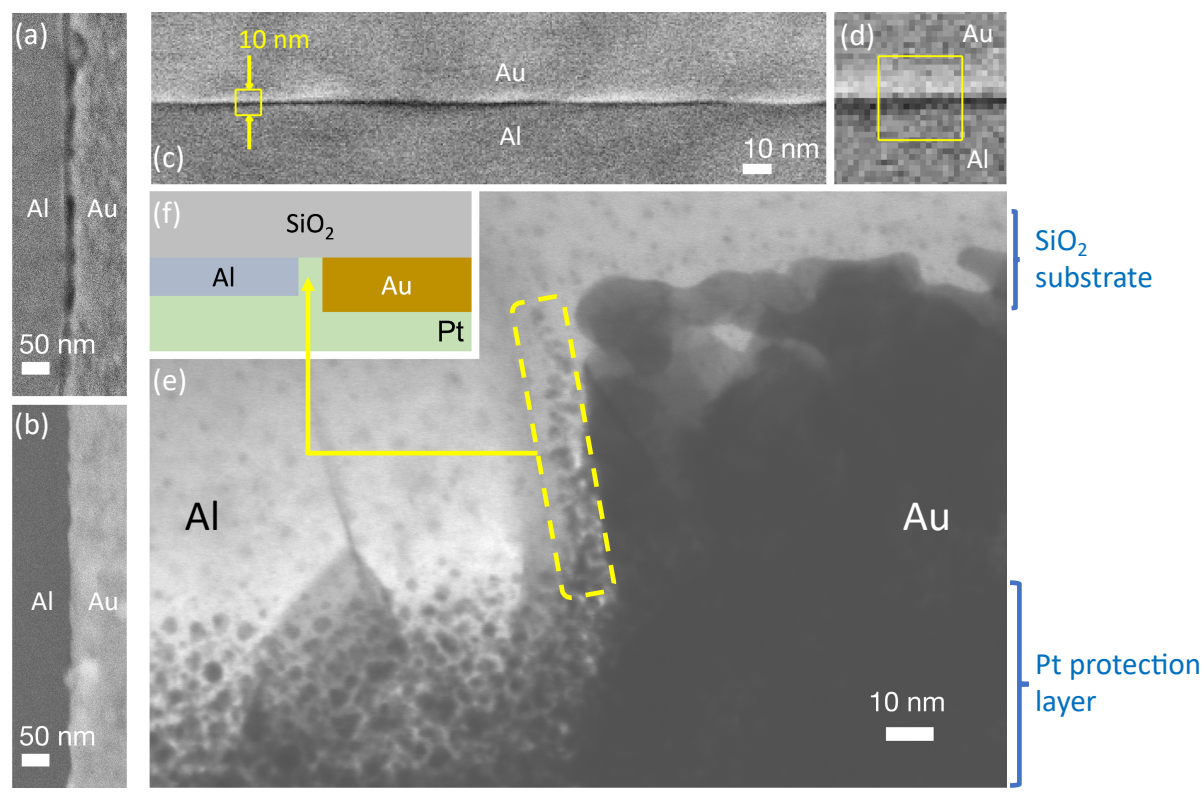

Figure 2. Images of nanogap electrodes formed using octadecane thiol as an adhesion modifier. (a, b) SEM images of an Au-Al nanogap obtained with matched (a) and unmatched (b) metal heights, using ODT as the SAM, 50-nm Au for M1 and 50-nm Al or 30-nm Al for M2. (The image contrast in (a) has been enhanced using contrast-limited adaptive histogram equalization for easier visualisation of the gap region). (c) SEM image showing a 240-nm section of a 50-nm-Au/30-nm-Al nanogap obtained using a high resolution S(T)EM. The yellow box indicates an area of 10-nm by $10-\mathrm{nm}$. (d) Magnified section of the SEM image from (c), showing the same 10-nm by 10-nm boxed region. (e) Transmission electron micrograph of the nanogap. The dotted yellow region shows platinum metal that has infiltrated the nanogap during sample preparation (see Methods). (f) Schematic representation of the TEM image in (e). 


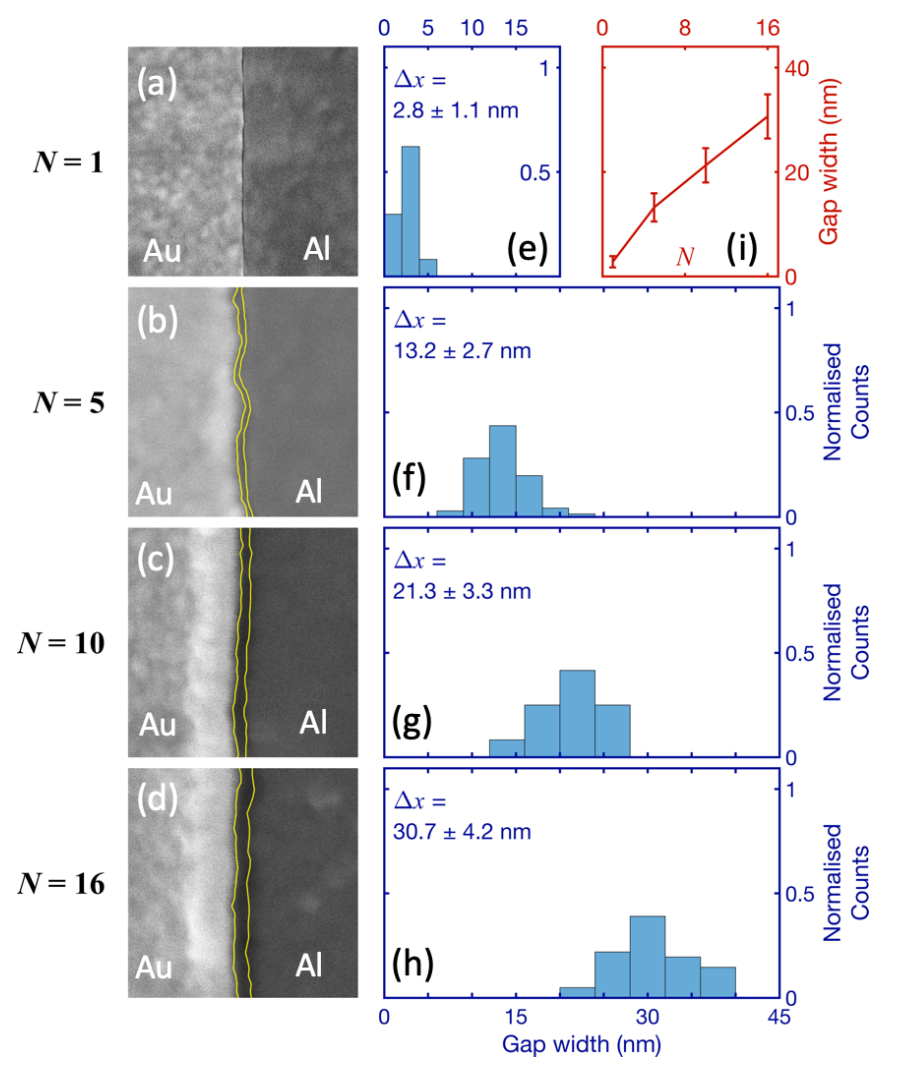

Figure 3. Representative scanning electron microscope images and corresponding spacing histograms for nanogaps obtained using size-tuneable adhesion lithography. (a-d) Scanning electron microscope images of 500-nm sections of $\mathrm{Au} / \mathrm{Al}$ nanogaps obtained using molecular rulers of length $N=1$ (a), $N=5$ (b), $N=10$ (c) and $N=16$ (d). The annotated yellow lines indicate the edges of the two metals. (e-h) Spacing histograms for the nanogaps in (a-d). The numerical values for the gapwidth $\Delta x$ represent the mean measured gap-width plus or minus one standard deviation. (i) Plot of the mean measured gapwidth versus the length $N$ of the molecular ruler. The length of each error bar is twice the standard deviation of the measured gap-width. 
(a)

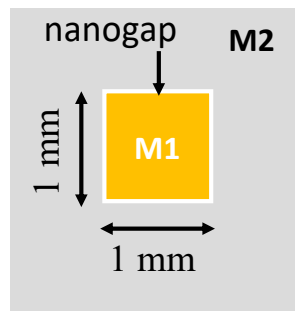

$N=1$

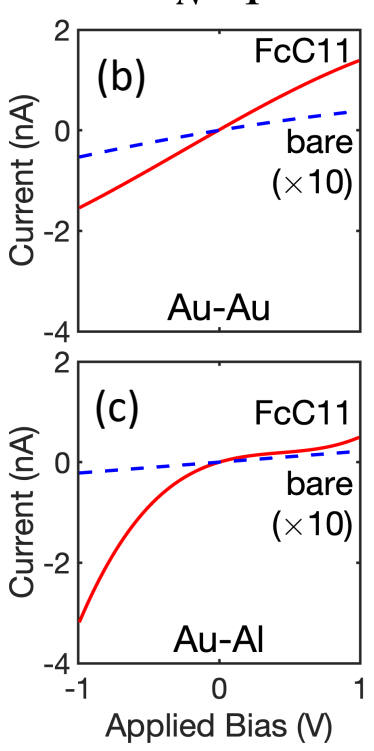

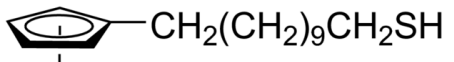

$\mathrm{Fe}$

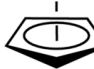

$\mathrm{M1}=\mathrm{Au}$

$\mathrm{M} 2$ = Au or Al

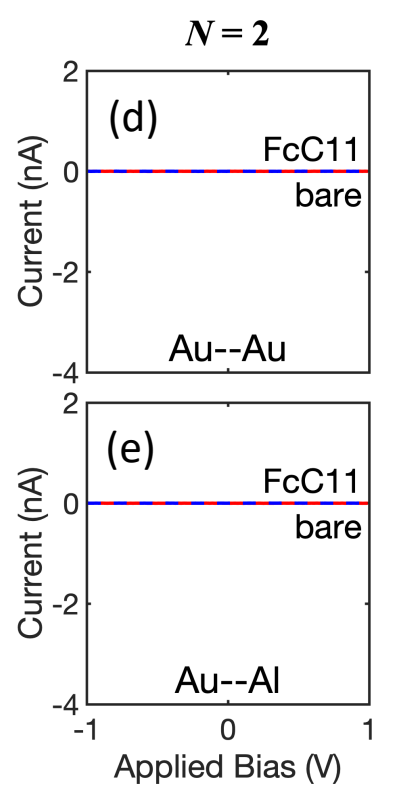

Figure 4. Current-voltage (I-V) characteristics for nanogap molecular diodes based on the conductive molecule FcC11. (a) Schematic showing the geometry of the nanogap electrodes used for the measurements, with chemical structure of ferrocenyl-1-undecanethiol (FcC11) shown adjacent. (b) $I-V$ characteristics for an Au-Au nanogap device fabricated using molecular rulers of length $N=1$, i.e. using a single layer of ODT. The dotted blue line and the solid red line show the $I-V$ characteristics before and after deposition of the FcC11 monolayer. (c) $I-V$ characteristics for an Au-Al nanogap device fabricated using molecular rulers of length $N=1$. (d) $I-V$ characteristics for an Au-Au nanogap device fabricated using molecular rulers of length $N=2$, i.e. using a bilayer of MHDA and ODT. The measured currents are below the $10 \mathrm{pA}$ detection limit of the current meter. (e) $I-V$ characteristics for an Au-Al nanogap device fabricated using molecular rulers of length $N=2$. The measured currents are below the $10 \mathrm{pA}$ detection limit. 

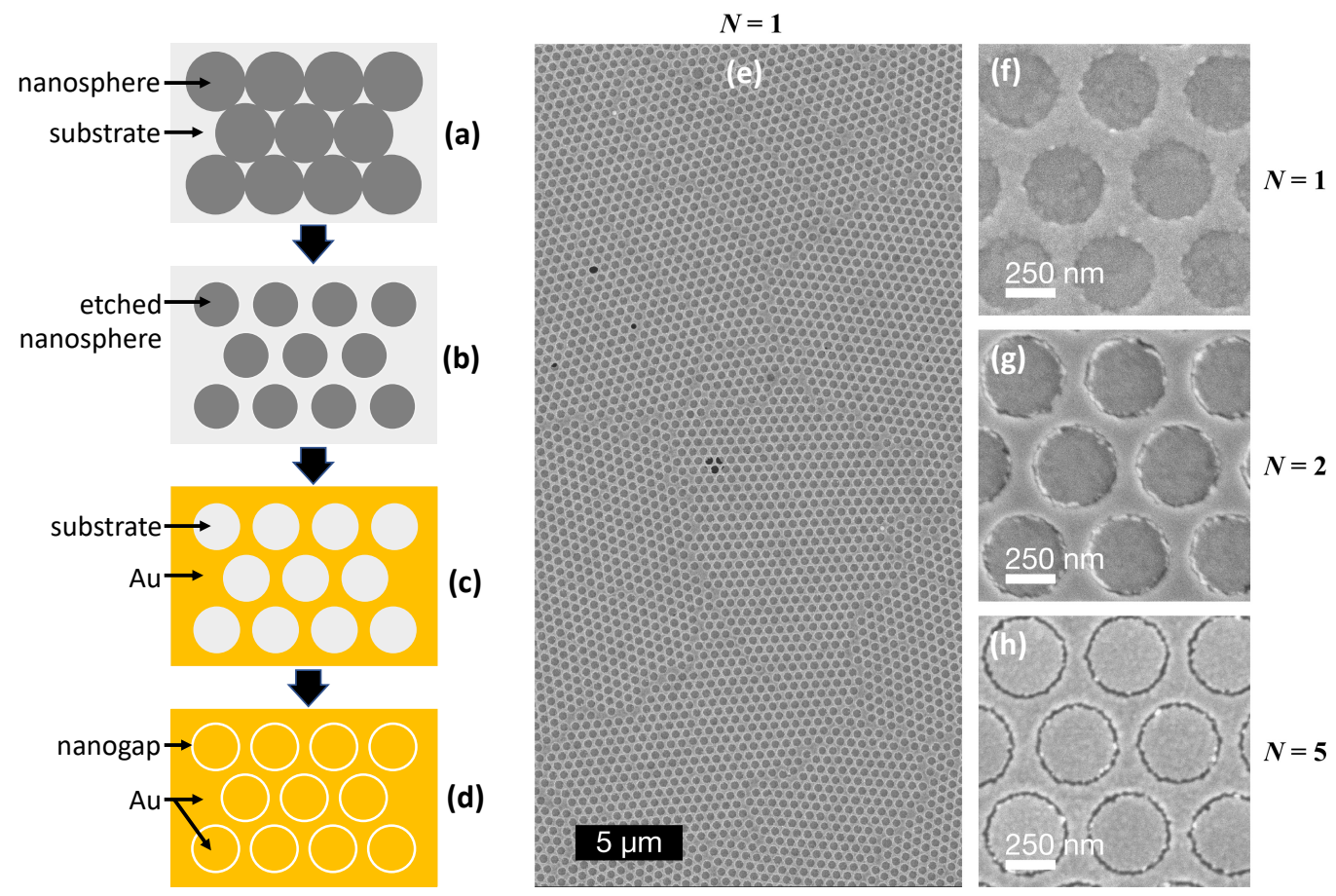

Figure 5. Fabrication of massively parallel nanoring arrays using a combination of nanosphere lithography and sizetuneable adhesion lithography. (a-d) Schematic of the fabrication procedure, in which: first, a monolayer of close-packed polystyrene nanospheres is deposited on a substrate (a); second, the nanospheres are "shrunk" by oxygen plasma treatment, leaving voids between them (b); third, metal $\mathrm{M} 1=\mathrm{Au}$ is deposited on the substrate through the nanosphere template and the template is removed, leaving a hexagonal array of nanoholes in the Au film (c); and, fourth, the holes are "filled" with a second metal $(\mathrm{M} 2=\mathrm{Au})$ using size-tuneable adhesion lithography, resulting in a hexagonal array of ring-shaped nanogaps (d). (e) $20-\mu \mathrm{m} \times 40-\mu \mathrm{m}$ SEM image of an Au-Au nanoring array, obtained using a molecular ruler of length $N=1$. The image shows several domains of nanorings with relatively low defect densities in each domain. $(\mathbf{f}-\mathbf{h})$ High magnification SEM images of Au-Au nanoring arrays, obtained using molecular rulers of length $N=1$ (f), $N=2$ (g) and $N=5$ (h). Each array has a pitch of $\sim 500 \mathrm{~nm}$ and a ring-diameter of $\sim 380 \mathrm{~nm}$, defined by the nanosphere diameters before and after etching. 
(a)

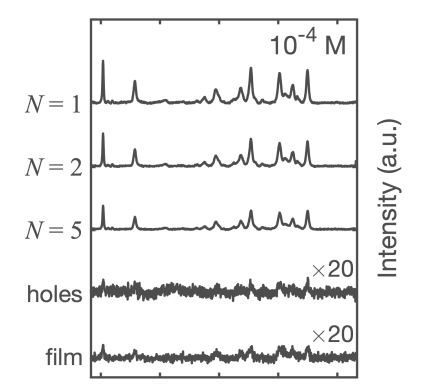

$0 \quad 500$
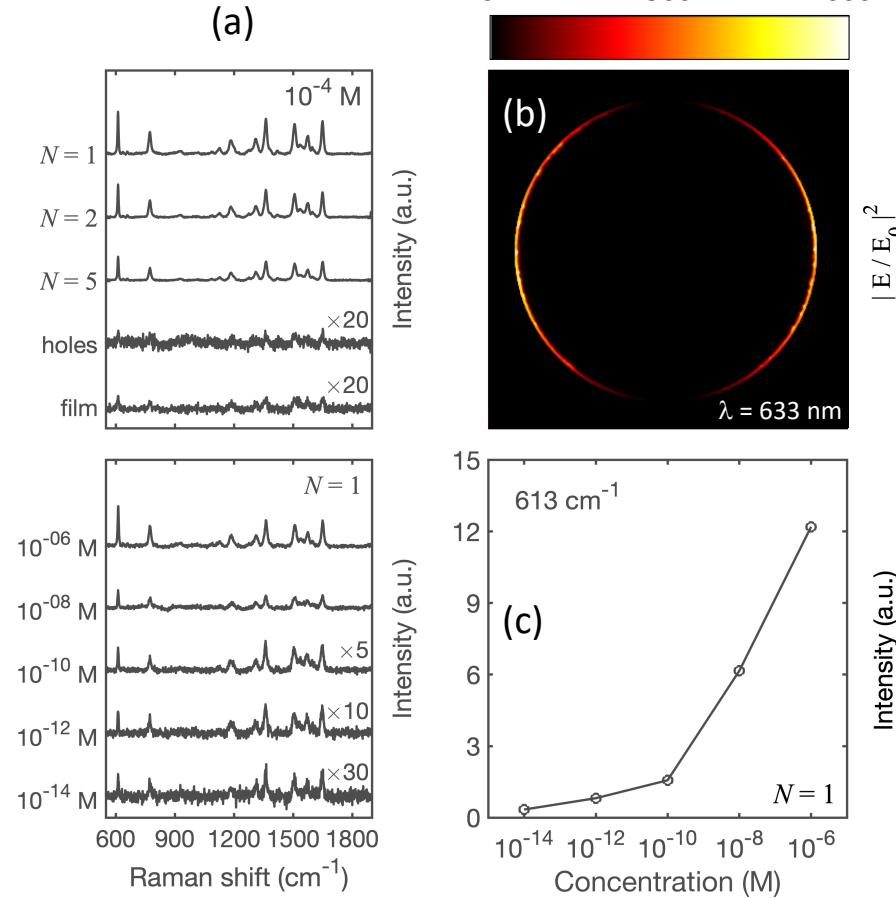

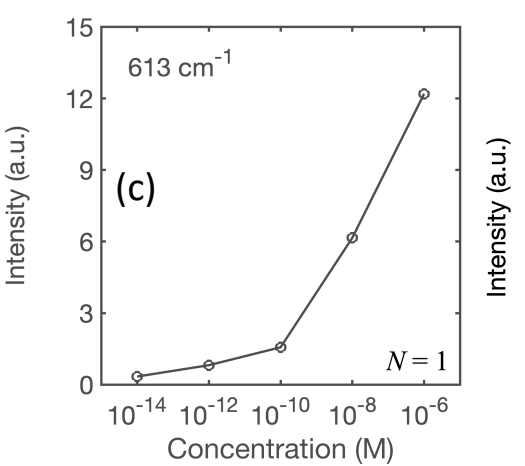

Figure 6. Surface-enhanced Raman scattering from arrays of ring-shaped nanogaps. (a, top) Experimentally determined Raman scattering spectra for Rhodamine 6G drop-cast from a $10^{-4} \mathrm{M}$ solution onto Au-Au RSN arrays fabricated using molecular rulers of length $N=1,2$ and 5 . The arrays were fabricated with a pitch of $\sim 500 \mathrm{~nm}$ and a ring-diameter of $\sim 380 \mathrm{~nm}$, see Fig. $5 \mathrm{f}$-h. Also shown for comparison are the Raman scattering spectra for $10^{-4} \mathrm{M}$ Rhodamine $6 \mathrm{G}$ drop-cast onto a thin gold film and onto a gold nanohole array, obtained by terminating the patterning procedure at step (c) of Fig. 5. All spectra were obtained under equivalent conditions with a $633 \mathrm{~nm}$ excitation wavelength, see Methods. The spectra for the thin gold film and the nanohole array have been multiplied by a factor of 20 for clarity. (a, bottom) Experimentally determined Raman scattering spectra for Rhodamine 6G drop-cast onto $N=1$ Au-Au RSN arrays from Rhodamine $6 \mathrm{G}$ dye solutions of varying concentration. All spectra were obtained under equivalent conditions with a $633 \mathrm{~nm}$ excitation wavelength. The spectra for $10^{-10} \mathrm{M}, 10^{-12} \mathrm{M}$ and $10^{-14} \mathrm{M}$ Rhodamine $6 \mathrm{G}$ have been multiplied by factors of 5, 10 and 30, respectively. (b) Simulated plot showing the square of the field enhancement at the height of the exposed metal surface for a ring-shaped nanogap in the hexagonal array, assuming a gap width of $\Delta r=3 \mathrm{~nm}(N=1)$. (c) Plot of experimentally determined scattering intensity at $613 \mathrm{~cm}^{-1}$ versus dye concentration, extracted from the data shown in the lower plot of (a). 
Fabrication of size-controlled metallic nanogaps down to the sub 3-nm level

Sihai Luo ${ }^{1}$, Andrea Mancini ${ }^{2}$, Rodrigo Berté ${ }^{2}$, Bård H. Hoff ${ }^{1}$, Stefan A. Maier ${ }^{2,3}$ and John C. deMello ${ }^{1 *}$

${ }^{1}$ Dept. Chemistry, Norwegian University of Science and Technology (NTNU), NO-7491 Trondheim, Norway

${ }^{2}$ Nano-Institute Munich, Faculty of Physics, Ludwig-Maximilians-Universität München, 80539 München, Germany

${ }^{3}$ Blackett Laboratory, Department of Physics, Imperial College London, London SW7 2AZ, UK

*john.demello@ntnu.no

\section{Supporting Information}



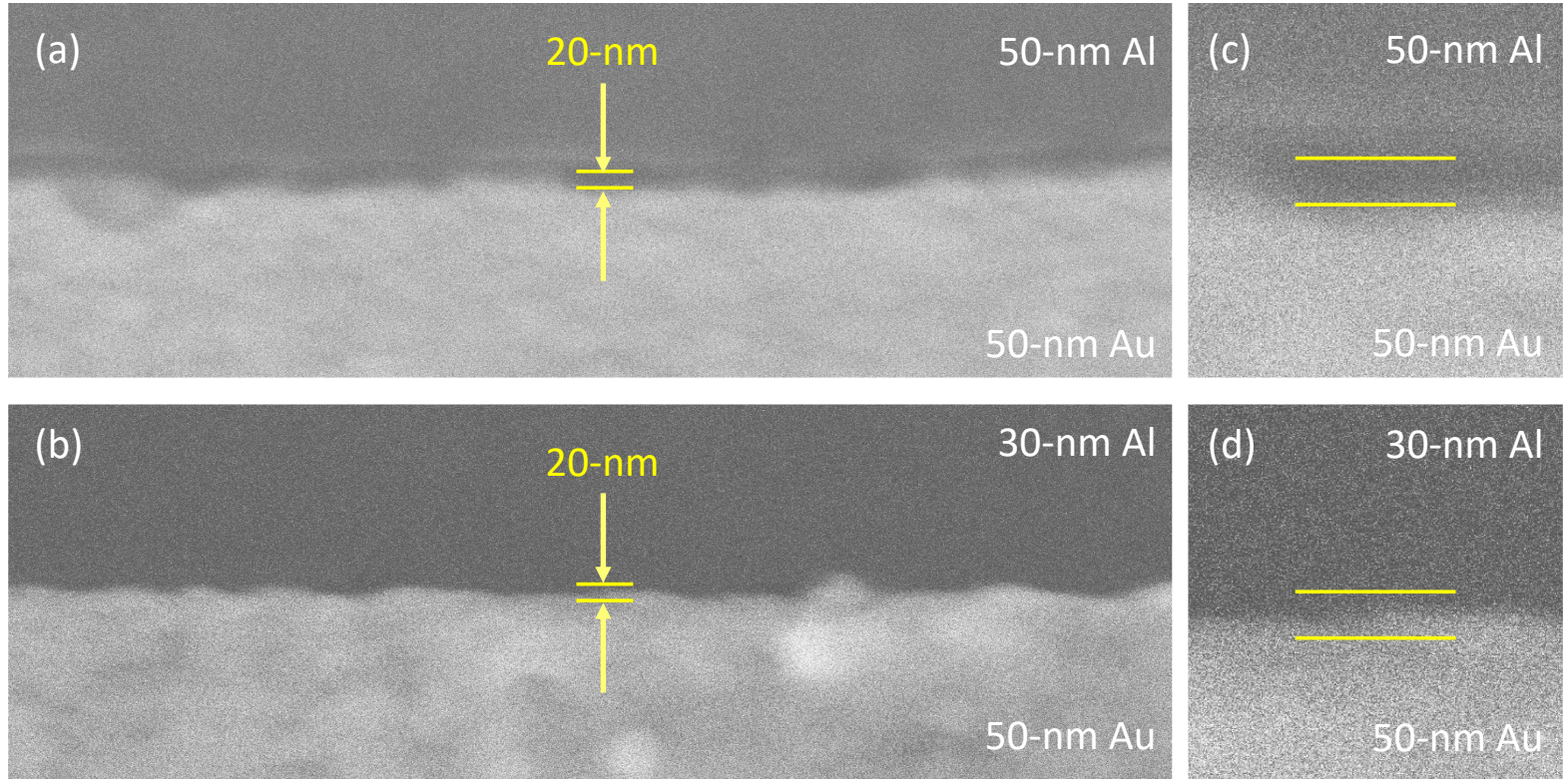

Figure S1. Effect of matched and unmatched metal heights on the gap-width. (a, b) SEM images of Au-Al nanogap electrodes obtained with matched (a) and unmatched (b) metal heights, using ODT as the SAM, 50-nm Au for M1 and either 50-nm Al (a) or 30-nm Al (b) for M2. The horizontal yellow lines are separated by $20 \mathrm{~nm}$. (c, d) Magnified images of the highlighted sections in $(a, b)$. Note, the images shown in this figure are magnified versions of the images in Fig. 2a,b (without contrast enhancement). The horizontal yellow lines are separated by $20 \mathrm{~nm}$. 


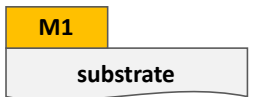

(a)

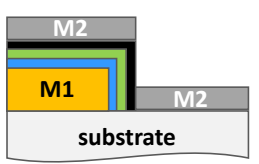

(e)

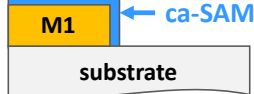

(b)

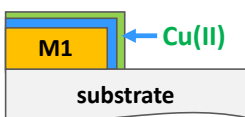

(c)

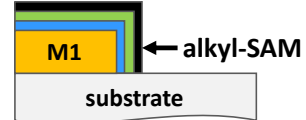

(d)

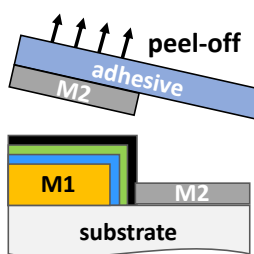

(f)

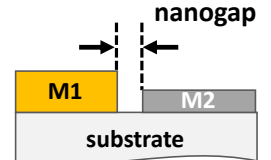

(g)

Figure S2. Schematic showing the key processing steps for size-tuneable adhesion lithography (a-g). First, metal M1 is deposited on a substrate and patterned as required (a). Second, M1 is selectively coated with a carboxylic acidfunctionalised metallophilic self-assembled monolayer (ca-SAM) (b). Third, the substrate is immersed in a solution of copper perchlorate, causing an atomically thin linker layer of copper ions to attach to the (outwardly facing) carboxylic acid groups on the ca-SAM molecules (c). The second and third-steps may be repeated as required to add further layers to the multilayer. Fourth, for the last layer in the assembly, the multilayer is capped with an alkyl-SAM (d). Fifth, metal M2 is deposited uniformly over the full area of the substrate at a substantially lower thickness than $\mathrm{M} 1$ (e). Sixth, an adhesive film is applied to the surface of $\mathrm{M} 2$ and peeled away from the substrate, selectively removing $\mathrm{M} 2$ from those regions located directly above the multilayer ( $f$ ). Lastly, the multilayer is removed by UV/ozone or oxygen-plasma treatment, leaving M1 and M2 sitting in a complementary arrangement side-by-side on the substrate (g), separated in the limiting case by the length of the multilayer. 
(a)

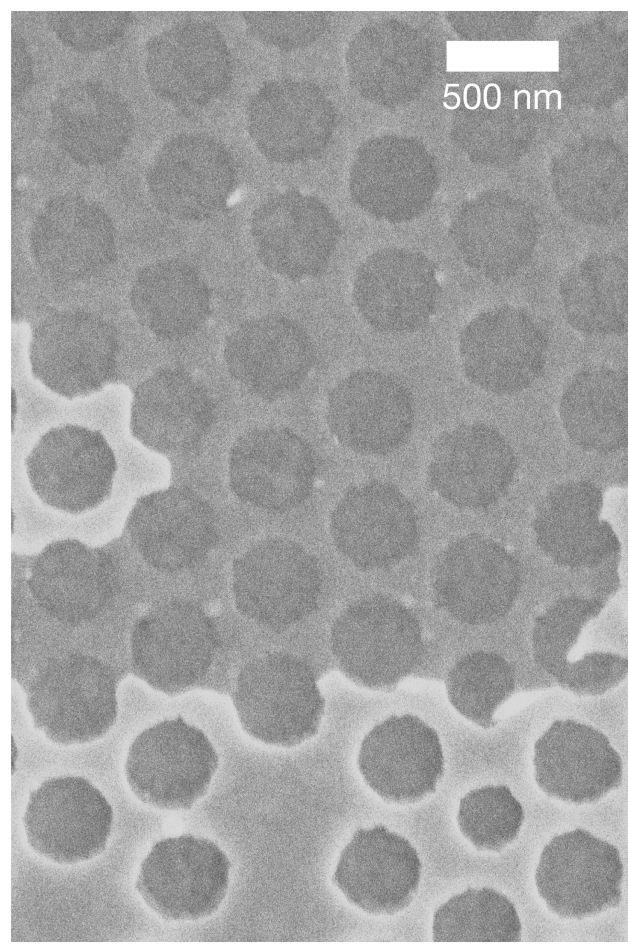

(b)

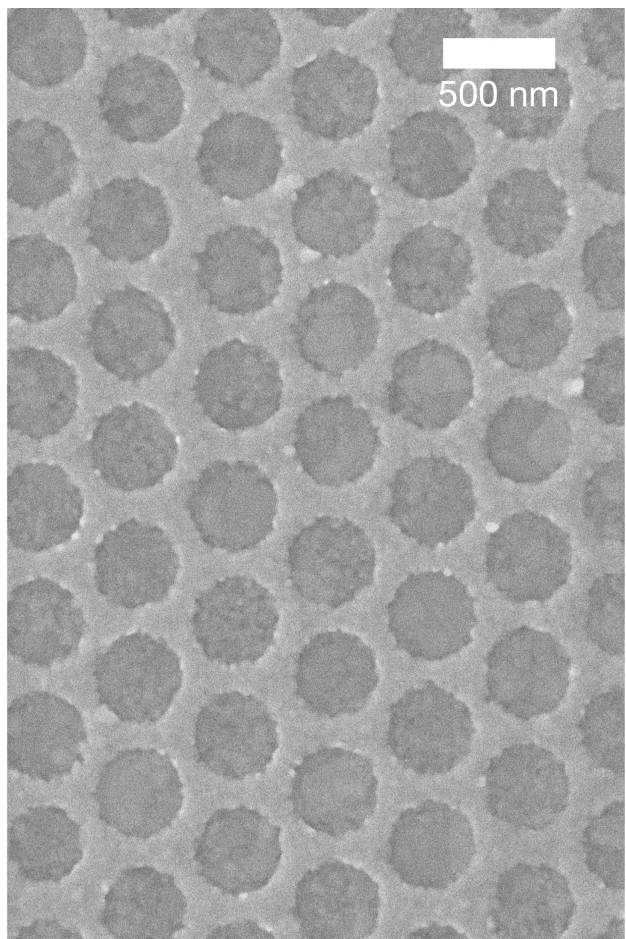

Figure S3. Effect of top-layer SAM on patterning quality. (a) Au/Au nanohole array obtained using MHDA as the top-layer, showing incomplete removal of the unwanted parts of the second metal M2. The bright regions correspond to unwanted gold that should have been stripped away but has instead been retained due to excessive adhesion between MHDA and $\mathrm{Au}$. (b) Equivalent Au/Au nanohole array obtained using ODT as the top-layer, showing successful removal of the unwanted parts of the second metal M2. Arrays have a pitch of $\sim 500 \mathrm{~nm}$ and a ring-diameter of $\sim 380 \mathrm{~nm}$, defined by the nanosphere diameters before and after etching. 


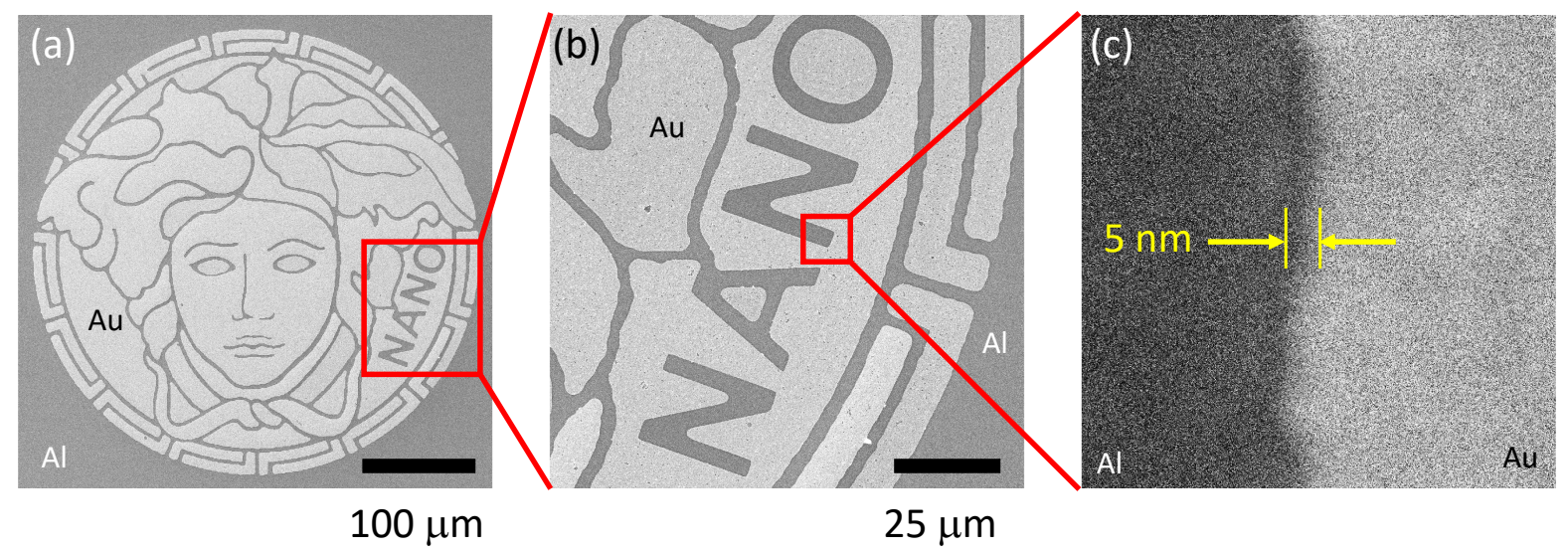

Figure S4. Multi-scale patterning by adhesion lithography. (a) Low magnification scanning electron micrograph of "Medusa", fabricated in gold and aluminium by adhesion lithography using a single layer of ODT. (b) Magnified section of the micrograph from (a). (c) Representative SEM image for a section of the Al/Au interface inside the red box in (b). A sub 5-nm nanogap exists at the interface between the two metals despite the full pattern extending over hundreds of microns. 
(a)

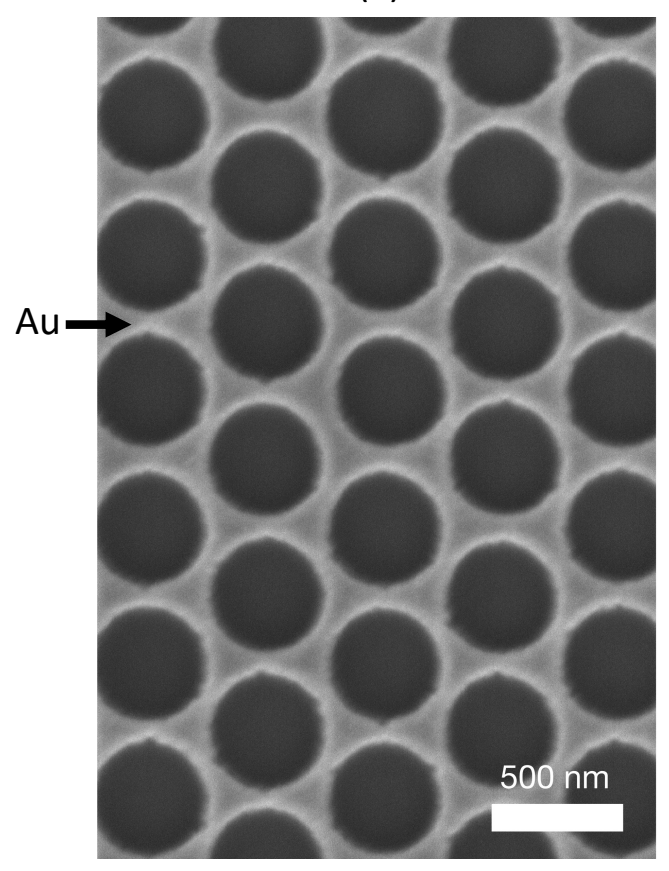

(b)

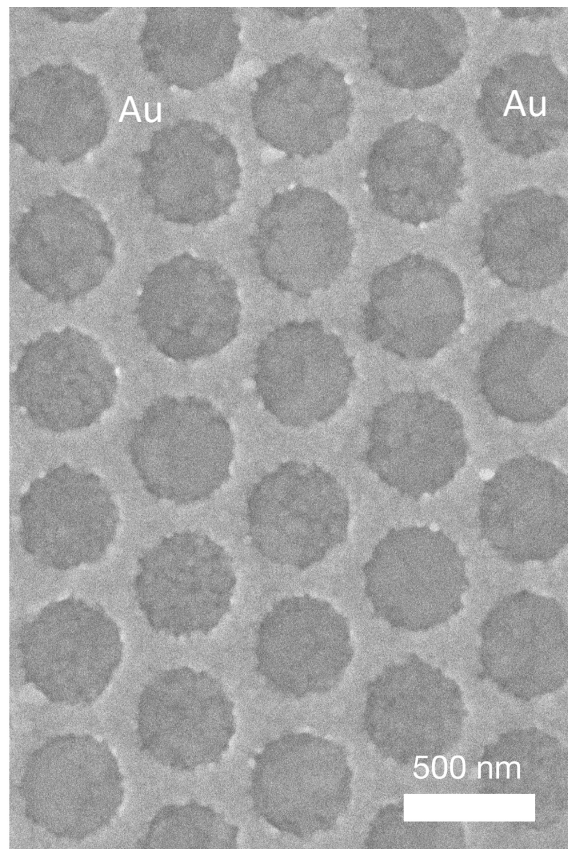

Figure S5. Nanohole and nanoring arrays, obtained using a combination of nanosphere lithography and adhesion lithography. (a) Nanohole array obtained by terminating fabrication after removal of the nanosphere template, i.e. at step (c) in Fig. 5 of the main article. Dark regions indicate the exposed substrate (glass). (b) Nanoring array obtained by carrying out the fabrication process to completion, i.e. to step (d) in Fig. 5. Arrays have a pitch of $\sim 500 \mathrm{~nm}$ and a ring-diameter of $\sim 380 \mathrm{~nm}$, defined by the nanosphere diameters before and after etching. A single layer of ODT was used as the adhesion modifier. 
(a)

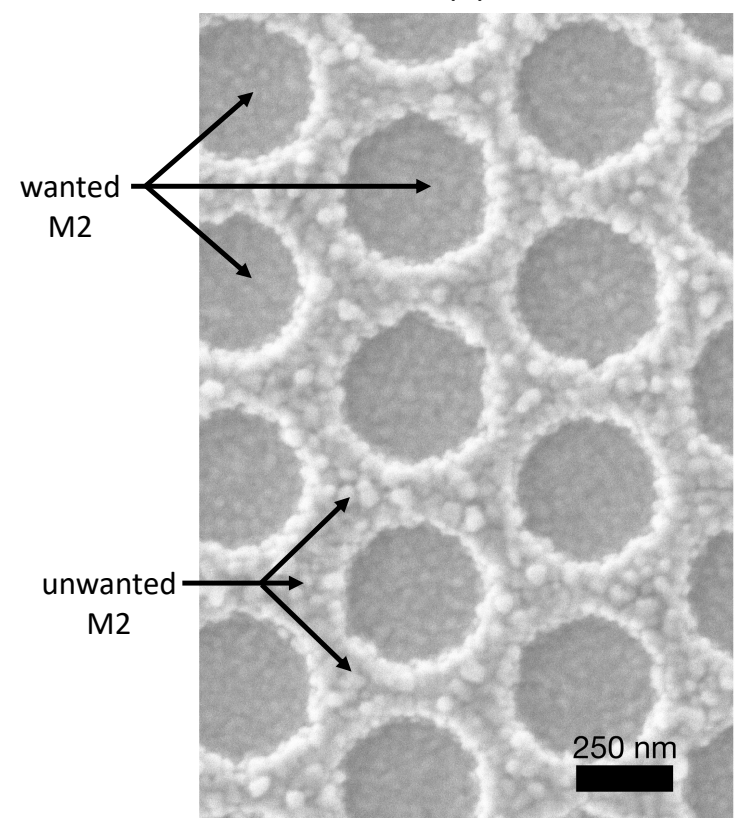

(b)

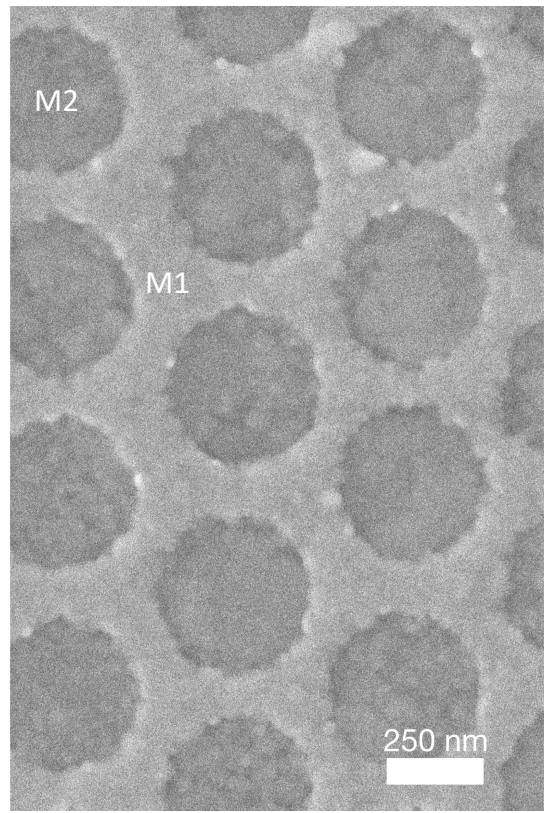

Figure S6. Au/Au nanoring arrays before and after the peeling step. Prior to peeling (a), a clear height difference is evident between the wanted (dark, recessed) and unwanted (bright, protruding) parts of $M 2$, causing pre-fracturing along the edge profile of M1. After peeling (b), M2 is recessed with respect to M1 due to its lower thickness. Arrays have a pitch of $\sim 500 \mathrm{~nm}$ and a ring-diameter of $\sim 380 \mathrm{~nm}$, defined by the nanosphere diameters before and after etching. ODT was used as an adhesion modifier. 
(a)

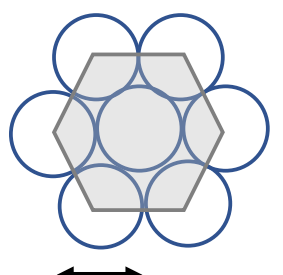

$2 r_{0}$ (b)

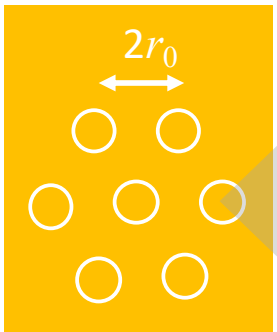

(c)

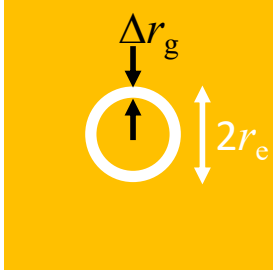

(d)

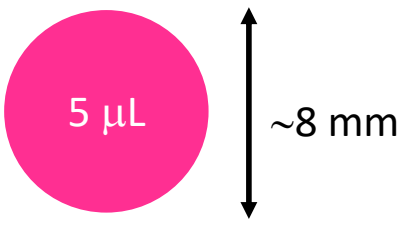

Figure S7. (a) Schematic showing polystyrene (PS) nanospheres of radius $r_{0}$ in a hexagonal close-packed arrangement. The hexagonal unit cell containing a total of three spheres is shown in grey. (b) Schematic of ring-shaped nanogap array, with a pitch $2 r_{0}$ that is determined by the original (unetched) radius $r_{0}$ of the PS nanospheres. (c) 'Close-up' schematic of individual nanoring of diameter $2 r_{e}$ and gap-width $\Delta r_{\mathrm{g}}$, where $r_{e}$ is the radius of the etched PS nanospheres and $\Delta r_{\mathrm{g}}$ is determined by the number of layers in the molecular ruler. (d) A 5- $\mu \mathrm{L}$ drop-cast dye solution fills a roughly circular spot of diameter $8 \mathrm{~mm}$. From simple geometry, the area $A$ of the hexagonal unit cell in (a) is given by $A=6 \sqrt{3} r_{0}^{2}$. Hence, the area - inclusive of voids $-A_{0}$ per sphere (or equivalently the area per ring in the final nanogap array) is one third this value, i.e. $A_{0}=2 \sqrt{3} r_{0}^{2}$. The approximate area $A_{\mathrm{g}}$ of each ring-shaped gap is given by $A_{g}=2 \pi r_{e} \Delta r_{g}$. Hence, the fraction $F$ of the array area that is covered by nanogaps is given by $F=A_{\mathrm{g}} / A_{0}=(\pi / \sqrt{3}) \Delta r_{g}\left(r_{e} / r_{0}^{2}\right)$. Putting $r_{0}=250 \mathrm{~nm}, r_{e}=$ $190 \mathrm{~nm}$ and $\Delta r_{g}=3 \mathrm{~nm}$, we obtain $A_{0}=216506 \mathrm{~nm}^{2}, A_{\mathrm{g}}=3581 \mathrm{~nm}^{2}$ and $F=1.65 \%$. A 5- $\mu \mathrm{L}$ drop of Rhodamine $6 \mathrm{G}$ dye solution deposited on the nanogap array forms a roughly circular spot of diameter $8 \mathrm{~mm}$, corresponding to an approximate area $A_{\text {dye }}=50 \mathrm{~mm}^{2}$ that covers around 230 million nanorings $\left(N_{\text {ring }}=A_{\text {dye }} / A_{0}\right)$. At the lowest detectable dye concentration of $c=10^{-14} \mathrm{M}$, a drop of volume $V=5 \mu \mathrm{L}$ contains around 30000 dye molecules $\left(N_{\mathrm{avg}} c V\right)$. For a random arrangement of dye molecules on top of the nanoring array, approximately 500 molecules are centred inside the nanogap $\left(F N_{\text {avg }} C V\right)$, implying the observed Raman scattering signal is due to of order 1000 dye molecules. 

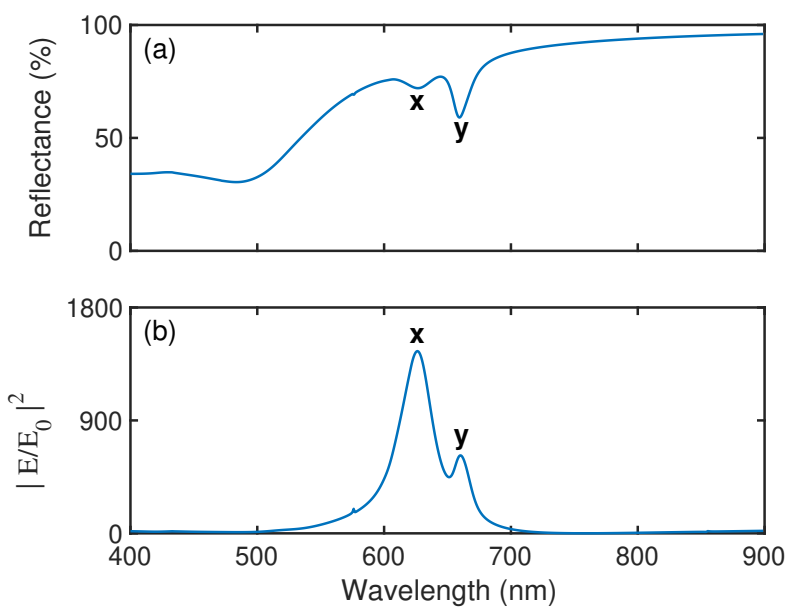

Figure S8. Simulation of the optical response of a Au/Au nanogap array with 3-nm gap-width. Simulated reflectance spectra (a) and field enhancement $\left(\left|\mathrm{E} / \mathrm{E}_{0}\right|^{2}\right)$ spectra (b), determined at the centre of the gap and level with the upper surface of M2. Calculations were carried out for an array pitch of $500 \mathrm{~nm}$, a ring diameter of $380 \mathrm{~nm}$, a gap-width of $3 \mathrm{~nm}$ and heights of 50 and $30 \mathrm{~nm}$ for M1 and M2, respectively. The dip in the reflectance spectrum at $\lambda=626 \mathrm{~nm}$ is due to the dipole mode arising across the edges of the gap, while the dip at $\lambda=660 \mathrm{~nm}$ is an array mode. The frequency of the gap resonance $(\mathbf{x})$ is mainly sensitive to the width of the gap, while the frequency of the array resonance $(\mathbf{y})$ is mainly sensitive to the pitch of the array. The drop in reflectivity for wavelengths below $600 \mathrm{~nm}$ is due to the interband transition of gold. Even though the array resonance gives rise to a larger dip in the reflectance spectrum, it can be seen from (b) that the gap resonance at $\lambda=626 \mathrm{~nm}$ is more effective at concentrating the electromagnetic energy inside the gap region, leading to higher SERS enhancement. The broad width of the gap-resonance results in strong SERS enhancement even when the excitation wavelength differs from the peak resonance by several tens of nanometres. 


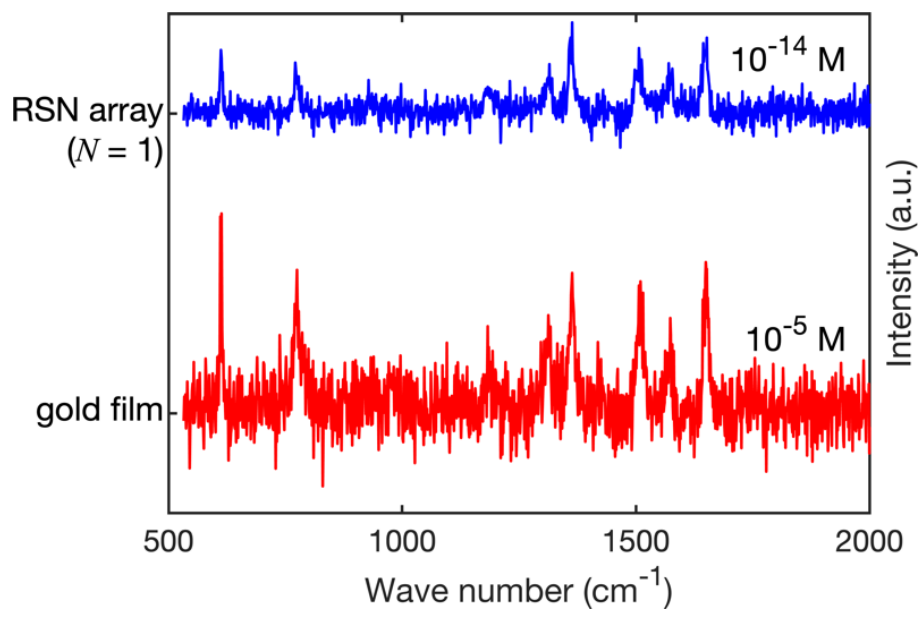

Figure S9. Determination of the Raman scattering enhancement factor relative to a thin gold film. The blue trace shows a Raman scattering spectrum for Rhodamine $6 \mathrm{G}$ drop-cast from a $10^{-14} \mathrm{M}$ solution onto an Au-Au RSN array with a pitch of $\sim 500 \mathrm{~nm}$ and a ring-diameter of $\sim 380 \mathrm{~nm}$, fabricated using a molecular ruler of length $N=1$ (see Fig. $5 \mathrm{f}$ ). The red trace shows a Raman scattering spectrum for Rhodamine $6 \mathrm{G}$ drop-cast from a $10^{-5} \mathrm{M}$ solution onto a $50-\mathrm{nm}$ gold film. 10-14 $\mathrm{M}$ and $10^{-5} \mathrm{M}$ represent the Raman scattering detection limits for the RSN array and the gold film, respectively. Spectra were obtained using identical acquisition parameters, see Methods. Following Ref. [S1], the analytical enhancement factor $\gamma$ at $613 \mathrm{~cm}^{-1}$ was determined using:

$$
\gamma=\frac{I_{\mathrm{RSN}}\left(613 \mathrm{~cm}^{-1}\right) / 10^{-14}}{I_{\mathrm{Au}}\left(613 \mathrm{~cm}^{-1}\right) / 10^{-5}}=\frac{0.3185 / 10^{-14}}{1.0 / 10^{-5}} \approx 3 \times 10^{8}
$$

[S1] Le Ru, E. C., Blackie, E., Meyer, M. \& Etchegoin, P. G. Surface enhanced raman scattering enhancement factors: A comprehensive study. J. Phys. Chem. C 111, 13794-13803 (2007). 